\title{
CIRJE-F-1165 \\ Portfolio Optimization with Choice of a Probability Measure
}

Taiga Saito

The University of Tokyo

Akihiko Takahashi

The University of Tokyo

April 2021; Revised in December 2021 and March 2022

CIRJE Discussion Papers can be downloaded without charge from:

http://www.cirje.e.u-tokyo.ac.jp/research/03research02dp.html

Discussion Papers are a series of manuscripts in their draft form. They are not intended for circulation or distribution except as indicated by the author. For that reason, Discussion Papers may not be reproduced or distributed without the written consent of the author. 


\section{Portfolio optimization with choice of a probability measure}

\author{
Taiga Saito \\ Graduate School of Economics \\ The University of Tokyo \\ Tokyo, Japan \\ staiga@e.u-tokyo.ac.jp
}

\author{
Akihiko Takahashi \\ Graduate School of Economics \\ The University of Tokyo \\ Tokyo, Japan \\ akihikot@e.u-tokyo.ac.jp
}

\begin{abstract}
This paper considers a new problem for portfolio optimization with a choice of a probability measure, particularly optimal investment problem under sentiments. Firstly, we formulate the problem as a sup-sup-inf problem consisting of optimal investment and a choice of a probability measure expressing aggressive and conservative attitudes of the investor. This problem also includes the case where the agent has conservative and neutral views on risks represented by Brownian motions and degrees of conservativeness differ among the risk. Secondly, we obtain an expression of the volatility process of a backward stochastic differential equation related to the conservative sentiment in order to investigate cases where the sup-sup-inf problem is solved. Specifically, we take a Malliavin calculus approach to solve the problem and obtain an optimal portfolio process. Finally, we provide an expression of the optimal portfolio under the sentiments in two examples with stochastic uncertainties in an exponential utility case and investigate the impact of the sentiments on the portfolio process.
\end{abstract}

Index Terms-Optimal portfolio problem, Uncertainty modeling, Malliavin calculus

\section{INTRODUCTION}

In this paper, we consider a new portfolio optimization problem with a choice of a probability measure, motivated by an investment problem under aggressive and conservative sentiments. When an agent who works on optimization on a control variable has an aggressive view on one risk, but has a conservative view on another risk at the same time, it is crucial to include those views in the optimization. To describe the views on different risks of an agent, Saito and Takahashi [19] introduced a sup-inf/inf-sup problem to represent the aggressive and conservative views on Brownian motions as a choice of probability measure and solved it by a BSDE approach. In Saito and Takahashi [19], the control variable for the optimization of the agent is exogenously given, and the attitudes towards different risks are formulated as a sup-inf/infsup problem on the views on the Brownian motions. This study further extends the approach and considers maximization on the control variable while choosing a probability measure that reflects the aggressive and conservative views on Brownian motions. Thus, we include the third optimization on the control variable in addition to a choice of a probability measure

This research is supported by CARF (Center for Advanced Research in Finance) and the grant from Tokio Marine Kagami Memorial Foundation. representing the sentiments under a given control variable and formulate the problem as a sup-sup-inf problem. Particularly, we take the infimum on the conservative view first to be more conservative on the expected utility. This study also includes the case where the agent has only conservative and neutral views on risks represented by Brownian motions, where degrees of conservativeness vary among the risks.

More concretely, we first set up an investor's investment problem under the sentiments as a combination of the optimal portfolio problem and a sup-inf problem representing the investor's sentiments. Then we propose the conditions under which the combined sup-sup-inf problem is solved. Particularly, we use a Malliavin calculus approach to obtain the conditions by obtaining the volatility term in a backward stochastic differential equation (BSDE). Finally, we obtain an optimal portfolio under the sentiments for an exponential utility case.

Investment under sentiments is an important issue for investors since the markets are often driven by not only economic conditions but also sentiments of the market participants. Specifically, it is often observed that the market is driven by sentiments, even when the economic environment deteriorates. Particularly, in the Japanese government bond market, while the interest rates were controlled and kept low by the central bank, the bond prices were determined by market sentiments. Nishimura et al. [14] and Nakatani et al. [13] estimated the sentiment in the market empirically by a text mining approach. In another instance, a home bias exists with which investors trade domestic assets aggressively while they trade foreign assets conservatively, since they know well about the domestic market but are not confident about the foreign market.

For related literature, Petersen et al. [17] consider stochastic uncertain systems where uncertainty is described by a constraint on the relative entropy. Benavoli and Chisci [2] deal with an optimal control problem for non-Gaussian discretetime linear stochastic systems with imprecise probabilities. Pun [18] investigates stochastic control problems for an ambiguity averse agent. In contrast to the robust control problems, our work incorporates not only the conservative side but also the aggressive side to consider optimal investment problems under sentiments since both conservative and aggressive sen- 
timents drive the financial markets. Moreover, we work on the combined two optimization problems, where we solve the optimal investment and aggressive and conservative sentiment problems. For applications of stochastic control to optimal portfolio problems, see Yiu et al. [26], Calafiore [4], [5], Mukuddem-Petersen and Petersen [12], Saito and Takahashi [20], [21], Ma et al. [11], Wang and Yu [24], Bensoussan et al. [3], Yan and Wong [25], Li et al. [10], and Dombrovskii et al. [7], for instance.

Specifically, Yiu et al. [26] consider an optimal portfolio problem under a maximum value-at-Risk constraint. Calafiore [4] proposes a data-driven approach for computing optimal portfolio compositions and [5] examines a multi-period sequential decision problems for financial asset allocation. Ma et al. [11] investigate an optimal portfolio execution problem under stochastic price impact and stochastic net demand pressure. Wang and $\mathrm{Yu}$ [24] consider a partial information non-zero-sum stochastic differential game of BSDEs with applications to an optimal portfolio problem. Yan and Wong [25] propose an open-loop control framework to time-consistent mean-variance portfolio problems in incomplete markets with stochastic volatility. Dombrovskii et al. [7] consider model predictive control for constrained discrete-time Markovian switching systems with an application to an optimal portfolio problem. Li et al. [10] examine a linear-quadratic problem for a time-delayed stochastic system with recursive utility with an application to a cash management problem. Mukuddem-Petersen and Petersen [12] propose a stochastic control problem for optimal risk management of banks. Saito and Takahashi [20] investigate an optimal trading problem of a hedger for derivatives pricing under liquidity costs and market impact, and [21] describe trading behaviors of different types of players in the high-frequency markets by a stochastic differential game. Bensoussan et al. [3] consider a non-zero-sum stochastic differential game for optimal investment and reinsurance problems between two insurance companies.

The organization of this paper is as follows. After setting up the financial market model, Section 3 describes the investor's investment problem under sentiments. Section 4 defines the sentiments of the investor as a choice of a probability measure. Section 5 describes the conditions under which the sup-supinf problem is solved. Section 6 presents the optimal portfolio under market sentiments. Finally, Section 7 concludes.

\section{Settings}

Let $(\Omega, \mathcal{F}, P)$ be a probability space. Let $\left\{B_{t}\right\}_{0 \leq t \leq T}$, $B_{t}=\left(B_{1, t}, \ldots, B_{d, t}\right)$, be a $d$-dimensional Brownian motion under $P$ and $\left\{\mathcal{F}_{t}\right\}_{0 \leq t \leq T}$ be the augmentation of the filtration generated by the Brownian motion $B$.

Firstly, we set up the financial markets as follows. Let $\left\{S_{0, t}\right\}_{0 \leq t \leq T}$ be a money market account process satisfying $d S_{0, t}=r_{t} S_{0, t} d t, \quad S_{0,0}=1$, where $r$ is a progressively measurable process satisfying $\int_{0}^{T} r_{s}^{2} d s<\infty, P-$ a.s. Also, let $\left\{S_{i, t}\right\}_{0 \leq t \leq T}, i=1, \ldots, d$ be price processes of risky assets following stochastic differential equations (SDEs) $d S_{i, t}=$ $b_{i, t} S_{i, t} d t+S_{i, t} \sum_{j=1}^{d} \sigma_{i, j, t} d B_{j, t}$, where $b_{i}, i=1, \ldots, d$ and $\sigma_{i, j}, 0 \leq i, j \leq d$ are progressively measurable processes satisfying $\int_{0}^{T}\left|b_{i, s}\right| d s<\infty, \int_{0}^{T} \sigma_{i, j, s}^{2} d s<\infty, P-a . s$. We assume no-arbitrage and completeness for the financial market, i.e. $\sigma_{t}=\left(\sigma_{i, j, t}\right)_{i, j=1, \ldots, d}$ is invertible for $0 \leq \forall t \leq T$, and there exists the unique market price of risk $-\theta$ such that $-\theta_{t}=\sigma^{-1}\left(\mathbf{b}_{t}-r_{t} \mathbf{1}\right)$, where $\mathbf{b}=\left(b_{1}, \ldots, b_{d}\right)^{\top}$ and $\mathbf{1}=(1, \ldots, 1)^{\top}$. Hereafter, we denote by $\top$ the transpose of a matrix. Let $Q$ be the risk neutral probability measure $Q$ defined as $\frac{d Q}{d P}=\mathcal{Z}_{T}=\exp \left(-\frac{1}{2} \int_{0}^{T}\left|\theta_{t}\right|^{2} d t+\int_{0}^{T} \theta_{t}^{\top} d B_{t}\right)$, and we define the state-price density process $H_{0}$ as $H_{0, t}=\frac{\mathcal{Z}_{t}}{S_{0, t}}$.

Moreover, let $\pi_{i}, i=1, \ldots, d$, be the value of position for the asset $i$ held by the investor, satisfying $\int_{0}^{T} \pi_{i, t}^{2} d t<\infty$, $P-a . s$. Also, let $\pi_{0}$ be the value of the money market account position for the investor and $x$ be a positive constant, which describes an initial endowment. Let $X^{\pi}$ be the investor's portfolio value process described as $X_{t}^{\pi}=x+$ $\int_{0}^{t}\left(r_{s} X_{s}^{\pi}+\sum_{i=1}^{d} \pi_{i, s}\left(b_{i, s}-r_{s}\right)\right) d s$

$+\sum_{i=1}^{d} \sum_{j=1}^{d} \int_{0}^{t} \pi_{i, s} \sigma_{i, j, s} d B_{j, s}$, where we assume that the portfolio process $\pi=\left(\pi_{0}, \pi_{1}, \ldots, \pi_{d}\right)^{\top}$ is self-financing: $X_{t}^{\pi}=\pi_{0, t}+\sum_{i=1}^{d} \pi_{i, t}$.

Furthermore, let $\mathcal{A}$ be a set of self-financing strategies $\pi=\left(\pi_{0}, \pi_{1}, \ldots, \pi_{d}\right)^{\top}$ satisfying $\int_{0}^{T}\left|\pi_{s}\right|^{2} d s<\infty, P-a . s$. and $\left\{H_{0, t} X_{t}^{\pi}\right\}_{0 \leq t \leq T}$ is a supermartingale under $P$ to preclude arbitrage opportunities.

\section{PortFolio OPTIMIZATION}

Firstly, we consider a portfolio optimization problem with respect to a terminal wealth $X_{T}$, given the density process for the investor's subjective probability measure.

Let $\Lambda_{j}$ be the set of progressively measurable processes $\lambda_{j}$ : $[0, T] \times \Omega \rightarrow \mathcal{R}^{d_{j}}, j=1,2, d_{1}+d_{2}=d$, satisfying $\left|\lambda_{j, k, t}\right| \leq$ $\left|\bar{\lambda}_{j, k, t}\right|, \quad k=1, \ldots, d_{j}, 0 \leq t \leq T$ where $\bar{\lambda}_{j, k}, j=1,2, k=$ $1, \ldots, d_{j}$ satisfy a weak version of Novikov's condition (e.g. see Corollary 3.5.14 in Karatzas and Shreve [9]) as in Saito and Takahashi [19]; there exists a partition of [0,T], $0=t_{0}<$ $t_{1}<\cdots<t_{N}=T$, such that $E\left[\exp \left(\frac{1}{2} \int_{t_{n-1}}^{t_{n}}\left|\bar{\lambda}_{s}\right|^{2} d s\right)\right]<$ $\infty$, for all $1 \leq n \leq N$, where $\bar{\lambda}=\left(\bar{\lambda}_{1}^{\top}, \bar{\lambda}_{2}^{\top}\right)^{\top}$. We remark that in the specific examples in Section VI and Appendix B, we further restrict $\Lambda_{1}$ and $\Lambda_{2}$ suitably so that the sup-sup-inf problem, which will be proposed in Problem 1 in Section IV, is solved.

We also assume the following conditions as in Pardoux and Rascanu [16]. Let $u: \mathcal{R} \rightarrow \mathcal{R}$ be a utility function, which is twice differentiable, concave and increasing, that is, $u^{\prime \prime}<$ $0, u^{\prime}>0$.

Let $p>1, n_{p}=1 \wedge(p-1)$ and $V_{t}=\int_{0}^{t}\left(\frac{1}{n_{p}}\left|\bar{\lambda}_{s}\right|^{2} d s\right)$. Assume that there exists $\delta>\frac{p}{p-1}$ such that for $q=\frac{p \delta}{p+\delta}$ and $n_{q}=1 \wedge(q-1)$,

$$
\left\{\begin{array}{l}
E\left[e^{p V_{T}}\left|u\left(X_{T}\right)\right|^{p}\right]<\infty, \\
E\left[\left(\int_{0}^{T}\left|\bar{\lambda}_{s}\right|^{2} d s\right)^{\frac{\delta}{2}}\right]<\infty, \\
E\left[\exp \left(\delta\left(\frac{1}{n_{q}}-\frac{1}{n_{p}}\right) \int_{0}^{T}\left|\bar{\lambda}_{s}\right|^{2} d s\right)\right]<\infty .
\end{array}\right.
$$

Also, let $\lambda=\left(\lambda_{1}^{\top}, \lambda_{2}^{\top}\right)^{\top}, \lambda_{j} \in \Lambda_{j}, j=1,2$, and $P^{\lambda_{1}, \lambda_{2}}$ be the investor's subjective probability measure defined by 
$\frac{d P^{\lambda_{1}, \lambda_{2}}}{d P}=\eta_{T}^{\lambda_{1}, \lambda_{2}}$ where $\eta_{t}^{\lambda_{1}, \lambda_{2}}=\exp \left(-\frac{1}{2} \int_{0}^{t}\left|\lambda_{s}\right|^{2} d s+\right.$ $\left.\int_{0}^{t} \lambda_{s}^{\top} d B_{s}\right)$. By Girsanov's theorem, $B_{t}^{\lambda_{1}, \lambda_{2}}$ defined as $B_{t}^{\lambda_{1}, \lambda_{2}}=B_{t}-\int_{0}^{t} \lambda_{s} d s$ is a Brownian motion under $P^{\lambda_{1}, \lambda_{2}}$. Since $d B_{t}=d B_{t}^{\lambda_{1}, \lambda_{2}}+\lambda_{t} d t, \lambda$ is considered to be biases on the view of the original Brownian motion under the investor's subjective measure $P^{\lambda_{1}, \lambda_{2}}$.

We consider maximization of the expected utility for the investor's terminal wealth $E^{P^{\lambda_{1}, \lambda_{2}}}\left[u\left(X_{T}^{\pi}\right)\right]$ over the strategies $\pi=\left(\pi_{0}, \pi_{1}, \ldots, \pi_{d}\right)^{\top} \in \mathcal{A}$ under the budget constraint

$$
E\left[H_{0, T} X_{T}^{\pi}\right] \leq x
$$

Then, it is well-known that the constrained maximization reduces to setting a Lagrangian multiplier $y>$ 0 and solving the unconstrained maximization problem: $\max _{\pi \in \mathcal{A}} E\left[\eta_{T}^{\lambda_{1}, \lambda_{2}} u\left(X_{T}^{\pi}\right)\right]+y\left[x-E\left[H_{0, T} X_{T}^{\pi}\right]\right]$, where the optimal solution is obtained by solving the first order condition is as follows. (See Theorem 3.7.6 in Karatzas and Shreve [8] for example).

$$
\eta_{T}^{\lambda_{1}, \lambda_{2}} u^{\prime}\left(X_{T}^{\pi}\right)=y H_{0, T}
$$

Then, the optimal terminal wealth $X_{T}^{*}$ is given as

$$
X_{T}^{*}=I\left(\frac{y H_{0, T}}{\eta_{T}^{\lambda_{1}, \lambda_{2}}}\right),
$$

where $I=\left(u^{\prime}\right)^{-1}$ and the Lagrangian multiplier $y>0$ is obtained as a unique solution of the equation

$$
x=E\left[H_{0, T} I\left(\frac{y H_{0, T}}{\eta_{T}^{\lambda_{1}, \lambda_{2}}}\right)\right] .
$$

\section{Conservative AND AGgRESSIVE SENTIMENTS}

Next, we express the investor's sentiment, which is conservative about the first Brownian motion $B_{1}$ and aggressive about the second Brownian motion $B_{2}$, as a sup-inf problem with respect to the expected utility of the terminal wealth given the terminal wealth $X_{T}^{\pi}$.

Let $J\left(\pi, \lambda_{1}, \lambda_{2}\right)=E^{P_{1}^{\lambda_{1}, \lambda_{2}}}\left[u\left(X_{T}^{\pi}\right)\right]$, and $\mathcal{S}^{0}$ be the space of $\mathcal{R}$-valued progressively measurable and continuous processes and $\Lambda_{d}^{0}$ be the space of progressively measurable processes $Z$ : $\Omega \times[0, T] \rightarrow \mathcal{R}^{d}$ such that $\int_{0}^{T}\left|Z_{t}\right|^{2} d t<+\infty, P-a . s$.

We consider the following sup-sup-inf problem.

\section{Problem 1.}

$$
\begin{aligned}
& \sup _{\pi \in \mathcal{A}} \sup _{\lambda_{2} \in \tilde{\Lambda}_{2}} \inf _{\lambda_{1} \in \Lambda_{1}} E^{P^{\lambda_{1}, \lambda_{2}}}\left[u\left(X_{T}^{\pi}\right)\right] \\
= & \sup _{\lambda_{2} \in \tilde{\Lambda}_{2}} \sup _{\pi \in \mathcal{A}} \inf _{\lambda_{1} \in \Lambda_{1}} E^{P^{\lambda_{1}, \lambda_{2}}}\left[u\left(X_{T}^{\pi}\right)\right] .
\end{aligned}
$$

We remark that in this problem, we take $\inf _{\lambda_{1} \in \Lambda}$ prior to $\sup _{\pi \in \mathcal{A}}$ and $\sup _{\lambda_{2} \in \tilde{\Lambda}_{2}}$ in order to consider the most conservative case on the expected utility.

Here, we assume that $\tilde{\Lambda}_{2}$ is a subset of $\Lambda_{2}$ such that for any $\lambda_{2} \in \tilde{\Lambda}_{2}$, there exists a saddle point $\left(\pi^{*}\left(\lambda_{2}\right), \lambda_{1}^{*}\left(\lambda_{2}\right)\right)$ such that (i) and (ii) below are satisfied. (i) For $\lambda_{1}^{*} \in \Lambda_{1}, \pi^{*} \in \mathcal{A}$ is the solution of the optimal portfolio problem, satisfying

$$
X_{T}^{\pi^{*}}=I\left(\frac{y H_{0, T}}{\eta_{T}^{\lambda_{1}^{*}, \lambda_{2}}}\right),
$$

where $y$ is a positive constant obtained as a solution of

$$
x=E\left[H_{0, T} I\left(\frac{y H_{0, T}}{\eta_{T}^{\lambda_{1}^{*}, \lambda_{2}}}\right)\right] .
$$

(ii) $\lambda_{1}^{*} \in \Lambda_{1}$ satisfies

$$
\lambda_{1, k, t}^{*}=-\left|\bar{\lambda}_{1, k, t}\right| \operatorname{sgn}\left(Z_{k, t}^{\pi^{*}, \lambda_{1}^{*}, \lambda_{2}}\right), k=1, \ldots, d_{1},
$$

where $\left(Y^{\pi^{*}, \lambda_{1}^{*}, \lambda_{2}}, Z^{\pi, \lambda_{1}^{*}, \lambda_{2}}\right) \in \mathcal{S}^{0} \times \Lambda_{d}^{0}$ is a unique solution of BSDE

$$
\begin{gathered}
Y_{t}^{\pi^{*}, \lambda_{1}^{*}, \lambda_{2}}= \\
-\left(\sum_{k=1}^{d_{1}}-\left|\bar{\lambda}_{1, k, t}\right|\left|Z_{k, t}^{\pi^{*}, \lambda_{1}^{*}, \lambda_{2}}\right|+\sum_{k=1}^{d_{2}} \lambda_{2, k, t} Z_{d_{1}+k, t}^{\pi^{*}, \lambda_{1}^{*}, \lambda_{2}}\right) d t \\
+\sum_{j=1}^{d_{1}+d_{2}} Z_{j, t}^{\pi^{*}, \lambda_{1}^{*}, \lambda_{2}} d B_{j, t}, Y_{T}^{\pi^{*}, \lambda_{1}^{*}, \lambda_{2}}=u\left(X_{T}^{\pi^{*}}\right) .
\end{gathered}
$$

Remark 1. We remark that by a slight modification of Theorem 1 in Saito and Takahashi [19], for given $\lambda_{2} \in \tilde{\Lambda}_{2}$ and $\pi^{*} \in \mathcal{A}, \lambda_{1}^{*} \in \Lambda_{1}$ that attains $\inf _{\lambda_{1} \in \Lambda_{1}} J\left(\pi^{*}, \lambda_{1}, \lambda_{2}\right)$, i.e. $J\left(\pi^{*}, \lambda_{1}^{*}, \lambda_{2}\right) \leq J\left(\pi^{*}, \lambda_{1}, \lambda_{2}\right), \forall \lambda_{1} \in \Lambda_{1}$ is given by (8) and $J\left(\pi^{*}, \lambda_{1}^{*}, \lambda_{2}\right)$ is expressed as $J\left(\pi^{*}, \lambda_{1}^{*}, \lambda_{2}\right)=Y_{0}^{\pi^{*}, \lambda_{1}^{*}, \lambda_{2}}$.

Also, we note that by Pardoux and Rascanu [16], BSDE (9) has a unique solution $\left(Y^{\pi^{*}, \lambda_{1}^{*}, \lambda_{2}}, Z^{\pi^{*}, \lambda_{1}^{*}, \lambda_{2}}\right) \in \mathcal{S}^{0} \times \Lambda_{d}^{0}$ such that $E\left(\sup _{t \in[0, T]} e^{p V_{t}}\left|Y_{t}^{\pi^{*}, \lambda_{1}^{*}, \lambda_{2}}\right|^{p}\right)<\infty$.

Then, the saddle point $\left(\pi^{*}\left(\lambda_{2}\right), \lambda_{1}^{*}\left(\lambda_{2}\right)\right)$ attains $\sup _{\pi \in \mathcal{A}} \inf _{\lambda_{1} \in \Lambda_{1}} J\left(\pi, \lambda_{1}, \lambda_{2}\right)$ for given $\lambda_{2} \in \tilde{\Lambda}_{2}$. This is observed as follows.

For an arbitrary $\pi \in \mathcal{A}$, let optimal $\lambda_{1, t}^{\pi}=$ $-\left|\bar{\lambda}_{1, t}\right| \operatorname{sgn}\left(Z_{1, t}^{\pi, \lambda_{1}, \lambda_{2}}\right)$.

Then, the saddle point $\left(\pi^{*}, \lambda_{1}^{*}\right)$ for given $\lambda_{2} \in \Lambda_{2}$ maximizes the expected utility as follows:

$$
\begin{aligned}
& E^{P_{1}^{\lambda_{1}^{\pi}, \lambda_{2}}}\left[u\left(X_{T}^{\pi}\right)\right]-E^{P_{1}^{\lambda_{1}^{*}, \lambda_{2}}}\left[u\left(X_{T}^{\pi^{*}}\right)\right] \\
& \leq E^{P_{1}^{\lambda_{1}^{*}, \lambda_{2}}}\left[u\left(X_{T}^{\pi}\right)-u\left(X_{T}^{\pi^{*}}\right)\right] \\
& \leq E^{P^{\lambda_{1}^{*}, \lambda_{2}}}\left[u^{\prime}\left(X_{T}^{\pi^{*}}\right)\left(X_{T}^{\pi}-X_{T}^{\pi^{*}}\right)\right] \\
& =E\left[\eta_{T}^{\lambda_{1}^{*}, \lambda_{2}} u^{\prime}\left(X_{T}^{\pi^{*}}\right)\left(X_{T}^{\pi}-X_{T}^{\pi^{*}}\right)\right] \\
& =y E\left[H_{0}(T)\left(X_{T}^{\pi}-X_{T}^{\pi^{*}}\right)\right] \leq y(x-x)=0 .
\end{aligned}
$$

Here, we used the optimality of $\lambda_{1}^{\pi} \in \Lambda_{1}$ for $\pi \in$ $\mathcal{A}$ in (10), the concavity of $u$ in (11), and the expression of $y$ (3) and the budget constraint (2) in (12). Thus $E^{P^{\lambda_{1}^{\pi}, \lambda_{2}}}\left[u\left(X_{T}^{\pi}\right)\right] \leq E^{P^{\lambda_{1}^{*}, \lambda_{2}}}\left[u\left(X_{T}^{\pi^{*}}\right)\right]$, which indicates $\sup _{\pi \in \mathcal{A}} \inf _{\lambda_{1} \in \Lambda_{1}} J\left(\pi, \lambda_{1}, \lambda_{2}\right)=J\left(\pi^{*}\left(\lambda_{2}\right), \lambda_{1}^{*}\left(\lambda_{2}\right), \lambda_{2}\right)$.

Therefore, the problem reduces to solve

$$
\sup _{\lambda_{2} \in \tilde{\Lambda}_{2}} J\left(\pi^{*}\left(\lambda_{2}\right), \lambda_{1}^{*}\left(\lambda_{2}\right), \lambda_{2}\right) .
$$


Remark 2. We can also show that the saddle point $\left(\pi^{*}\left(\lambda_{2}\right), \lambda_{1}^{*}\left(\lambda_{2}\right)\right)$ attains $\sup _{\pi \in \mathcal{A}} \inf _{\lambda_{1} \in \Lambda_{1}}$ for given $\lambda_{2} \in \tilde{\Lambda}_{2}$ as follows.

Since $J\left(\pi, \lambda_{1}^{*}\left(\lambda_{2}\right), \lambda_{2}\right) \leq J\left(\pi^{*}\left(\lambda_{2}\right), \lambda_{1}^{*}\left(\lambda_{2}\right), \lambda_{2}\right)$

$\leq J\left(\pi^{*}\left(\lambda_{2}\right), \lambda_{1}, \lambda_{2}\right), \quad \forall \lambda_{1} \in \Lambda_{1}, \pi \in \mathcal{A}$, we have $\inf _{\lambda_{1} \in \Lambda_{1}} \sup _{\pi \in \mathcal{A}} J\left(\pi, \lambda_{1}, \lambda_{2}\right) \leq J\left(\pi^{*}\left(\lambda_{2}\right), \lambda_{1}^{*}\left(\lambda_{2}\right), \lambda_{2}\right) \leq$ $\sup _{\pi \in \mathcal{A}} \inf _{\lambda_{1} \in \Lambda_{1}} J\left(\pi, \lambda_{1}, \lambda_{2}\right)$. Combining with the inequality $\sup _{\pi \in \mathcal{A}} \inf _{\lambda_{1} \in \Lambda_{1}} J\left(\pi, \lambda_{1}, \lambda_{2}\right) \leq J\left(\pi^{*}\left(\lambda_{2}\right), \lambda_{1}^{*}\left(\lambda_{2}\right), \lambda_{2}\right) \leq$ $\inf _{\lambda_{1} \in \Lambda_{1}} \sup _{\pi \in \mathcal{A}} J\left(\pi, \lambda_{1}, \lambda_{2}\right)$, which naturally holds, we obtain $J\left(\pi^{*}\left(\lambda_{2}\right), \lambda_{1}^{*}\left(\lambda_{2}\right), \lambda_{2}\right)=\sup _{\pi \in \mathcal{A}} \inf _{\lambda_{1} \in \Lambda_{1}} J\left(\pi, \lambda_{1}, \lambda_{2}\right)$ $\left(=\inf _{\lambda_{1} \in \Lambda_{1}} \sup _{\pi \in \mathcal{A}} J\left(\pi, \lambda_{1}, \lambda_{2}\right)\right)$. As this indicates, $\sup _{\pi \in \mathcal{A}}$ and $\inf _{\lambda_{1} \in \Lambda_{1}}$ are interchangeable. However, $\inf _{\lambda_{1} \in \Lambda_{1}}$ and $\sup _{\lambda_{2} \in \tilde{\Lambda}_{2}}$ cannot be exchanged. In this work, we consider taking inf in $_{\lambda_{1} \in \Lambda_{1}}$ first so that it becomes the most conservative case.

\section{A. Explicit expression of $Z^{\pi^{*}, \lambda_{1}^{*}, \lambda_{2}}$}

As we observed, finding $\left(\pi^{*}\left(\lambda_{2}\right), \lambda_{1}^{*}\left(\lambda_{2}\right)\right)$ that satisfies (i) and (ii) for given $\lambda_{2} \in \tilde{\Lambda}_{2}$ is crucial in solving the problem. As we will observe in the examples in Section VI and Appendix $\mathrm{B}$, we can take the following procedure to find the pair.

Step 1. Firstly, we suppose some specific form for $\lambda_{1}^{*}$ ( $\lambda_{1}^{*}=\bar{\lambda}_{1}$, for instance). More concretely, we predetermine $\operatorname{sgn}\left(Z_{k}^{\pi^{*}, \lambda_{1}^{*}, \lambda_{2}}\right), \quad k=1, \ldots, d_{1}$ in (8) as functional of some exogenously given random variable $\left(\operatorname{sgn}\left(Z_{k}^{\pi^{*}, \lambda_{1}^{*}, \lambda_{2}}\right)=\right.$ $-\operatorname{sgn}\left(\bar{\lambda}_{k}\right)$ for instance, as we will observe in the examples in Section VI and Appendix B).

Step 2. Then, we calculate $Z_{k}^{\pi^{*}, \lambda_{1}^{*}, \lambda_{2}}$ in (9) with $X_{T}^{\pi^{*}}$ given by (6).

Step 3. Finally, we confirm that $\operatorname{sgn}\left(Z_{k}^{\pi^{*}, \lambda_{1}^{*}, \lambda_{2}}\right)=\operatorname{sgn}\left(\bar{\lambda}_{k}\right)$ as predetermined. Then, $\pi^{*}$ generating $X_{T}^{\pi^{*}}$ and $\lambda_{1}^{*}$ constitute the pair.

In Section VI, we will provide examples where the saddle point exists for any $\lambda_{2} \in \tilde{\Lambda}_{2}$ and the sup-sup-inf problem is solved.

In the following, for given $\lambda_{1}^{*} \in \Lambda_{1}$, we provide an expression of $Z^{\pi^{*}, \lambda_{1}^{*}, \lambda_{2}}$ in BSDE (9) with $X_{T}^{\pi^{*}}$ in the terminal condition given by (6). Hereafter, we suppose $r \equiv 0$ for simplicity. See Appendices for general random cases for $r$.

Following the definitions of the Malliavin derivative operator and the Sobolev space in Ocone and Karatzas [15], we let $D_{t}$ be the Malliavin derivative operator with respect to $B_{t}$. $L_{1,1}^{a}$ denotes the set of $\mathcal{R}^{d}$-valued progressively measurable processes $\left\{v_{t}\right\}_{0 \leq t \leq T}$ such that (i) for almost every $s \in[0, T]$, $v(s, \cdot) \in\left(\mathbf{D}_{1,1}\right)^{d}$, (ii) $D v(s, \omega)=\left(D_{i} v_{j}(s, \omega)\right)_{i, j=1, \ldots, d} \in$ $\left(L^{2}[0, T]\right)^{d^{2}}$ admits a progressively measurable version, (iii) $E\left[\left(\int_{0}^{T}\left|v_{s}\right|^{2} d s\right)^{\frac{1}{2}}+\left(\int_{0}^{T}\|D v(s)\|^{2} d s\right)^{\frac{1}{2}}\right]<\infty$, where $\mathbf{D}_{1,1}$ denotes the Sobolev space and $\|\cdot\|$ denotes the $L^{2}([0, T])$ norm, i.e., $\|D v(s)\|^{2}=\int_{0}^{T} \sum_{i, j=1}^{d}\left|D_{i, t} v_{j}(s)\right|^{2} d t$.

Theorem 1. Let $\tilde{\lambda}=\left(\lambda_{1}^{* \top}, \lambda_{2}^{\top}\right)^{\top},\left(\lambda_{1}^{*}, \lambda_{2}\right) \in \Lambda_{1} \times \Lambda_{2}$ and $X_{T}^{\pi^{*}}$ be defined by (4) and (5) with $\eta=\eta^{\lambda_{1}^{*}, \lambda_{2}}$. Also, let $B^{\lambda_{1}^{*}, \lambda_{2}}$ be $P^{\lambda_{1}^{*}, \lambda_{2}}$-Brownian motion defined by $B_{t}^{\lambda_{1}^{*}, \lambda_{2}}=B_{t}-$ $\int_{0}^{t} \tilde{\lambda}_{s} d s$. We assume $\theta, \tilde{\lambda} \in L_{1,1}^{a}$. Then $Z^{\pi^{*}, \lambda_{1}^{*}, \lambda_{2}}$ in BSDE (9) has the following expression if it is well-defined.

$$
\begin{gathered}
Z_{i, t}^{\pi^{*}, \lambda_{1}^{*}, \lambda_{2}}=E^{P^{\lambda_{1}^{*}, \lambda_{2}}}\left[-I^{\prime}\left(\frac{y H_{0, T}}{\eta_{T}^{\lambda_{1}^{*}, \lambda_{2}}}\right)\left(\frac{y H_{0, T}}{\eta_{T}^{\lambda_{1}^{*}, \lambda_{2}}}\right)^{2}\right. \\
\left(+\left(\tilde{\lambda}_{i, t}-\theta_{i, t}\right)-\int_{t}^{T}\left(D_{i, t} \theta_{s}\right)\left(\tilde{\lambda}_{i, s}-\theta_{i, s}\right) d s\right. \\
\left.+\sum_{j=1}^{d} \int_{t}^{T} D_{i, t}\left(\tilde{\lambda}_{j, s}-\theta_{j, s}\right) d B_{j, s}^{\lambda_{1}^{*}, \lambda_{2}}\right) \\
\left.+u\left(X_{T}^{\pi^{*}}\right) \sum_{j=1}^{d} \int_{t}^{T} D_{i, t} \tilde{\lambda}_{j, s} d B_{j, s}^{\lambda_{1}^{*}, \lambda_{2}} \mid \mathcal{F}_{t}\right] .
\end{gathered}
$$

Moreover, when $X_{T}^{*}$ is $\mathcal{F}_{T}^{\lambda_{1}^{*}, \lambda_{2}}$-measurable, where $\left\{\mathcal{F}_{t}^{\lambda_{1}^{*}, \lambda_{2}}\right\}_{0<t<T}$ is the augmentation of the filtration generated by $B^{\lambda_{1}^{*}, \lambda_{2}}$, we have a more concise expression for $Z_{t}^{\lambda_{1}^{*}, \lambda_{2}}$. The following expression will be used in the examples in Section VI and Appendix B. Let $D_{t}^{\lambda_{1}^{*}, \lambda_{2}}$ be the Malliavin derivative operator with respect to $B_{t}^{\lambda_{1}^{*}, \lambda_{2}}$. Also, let $\mathbf{D}_{1,1}^{\lambda_{1}^{*}, \lambda_{2}}$ be the Sobolev space and $L_{1,1}^{a, \lambda_{1}^{*}, \lambda_{2}}$ be the set of $\mathcal{R}^{d}$-progressively measurable processes corresponding to $B^{\lambda_{1}^{*}, \lambda_{2}}$.

Corollary 1. In particular, if $X_{T}^{\pi^{*}} \in \mathbf{D}_{1,1}^{\lambda_{1}^{*}, \lambda_{2}}$ and $\tilde{\lambda}, \theta \in$ $L_{1,1}^{a, \lambda_{1}^{*}, \lambda_{2}}, Z^{\lambda_{1}^{*}, \lambda_{2}}$ has the following expression if it is welldefined.

$$
\begin{gathered}
Z_{i, t}^{\pi^{*}, \lambda_{1}^{*}, \lambda_{2}}=E^{P^{\lambda_{1}^{*}, \lambda_{2}}}\left[-I^{\prime}\left(\frac{y H_{0, T}}{\eta_{T}^{\lambda_{1}^{*}, \lambda_{2}}}\right)\left(\frac{y H_{0, T}}{\eta_{T}^{\lambda_{1}^{*}, \lambda_{2}}}\right)^{2}\right. \\
\left(\left(\tilde{\lambda}_{i, t}-\theta_{i, t}\right)+\frac{1}{2} \int_{t}^{T} D_{i, t}^{\lambda_{1}^{*}, \lambda_{2}}\left|\tilde{\lambda}_{s}-\theta_{s}\right|^{2} d s\right. \\
\left.\left.+\sum_{j=1}^{d} \int_{t}^{T} D_{i, t}^{\lambda_{1}^{*}, \lambda_{2}}\left(\tilde{\lambda}_{j, s}-\theta_{j, s}\right) d B_{j, s}^{\lambda_{1}^{*}, \lambda_{2}}\right) \mid \mathcal{F}_{t}^{\lambda_{1}^{*}, \lambda_{2}}\right] .
\end{gathered}
$$

Specifically, in the case of an exponential utility with $u(x)=$ $-\frac{1}{p} \exp (-p x), p>0$,

$$
\begin{aligned}
& Z_{i, t}^{\pi^{*}, \lambda_{1}^{*}, \lambda_{2}}=\frac{1}{p}\left(\frac{y H_{0, t}}{\eta_{t}^{\lambda_{1}^{*}, \lambda_{2}}}\right) E^{Q}\left[\left(\tilde{\lambda}_{i, t}-\theta_{i, t}\right)\right. \\
& \left.+\sum_{j=1}^{d} \int_{t}^{T} D_{i, t}^{\lambda_{1}^{*}, \lambda_{2}}\left(\tilde{\lambda}_{j, s}-\theta_{j, s}\right) d B_{j, s}^{Q} \mid \mathcal{F}_{t}^{\lambda_{1}^{*}, \lambda_{2}}\right] .
\end{aligned}
$$

Proof. By applying Theorem 2.5 in Ocone and Karatzas [15] to $u\left(X_{T}^{\pi^{*}}\right)$, we obtain the result. See Appendix A for details.

\section{OPTIMAL PORTFOLIO UNDER CONSERVATIVE AND AGGRESSIVE SENTIMENTS}

Finally, we provide an explicit expression of the portfolio process $\pi^{*}$ that generates the optimal terminal wealth $X_{T}^{\pi^{*}}$ under conservative and aggressive sentiments. Hereafter, we denote by $\left\{\mathcal{F}_{t}^{Q}\right\}_{0 \leq t \leq T}$ the augmentation of the filtration generated by the Brownian motion $B^{Q}$ under $Q$ and let $\mathbf{D}_{1,1}^{Q}$ be the Sobolev space and $L_{1,1}^{a, Q}$ be the $\mathcal{R}^{d}$-progressively 
measurable processes corresponding to $B^{Q}$. Also, let $D_{t}^{Q}$ be the Malliavin derivative with respect to the Brownian motion $B^{Q}$ under $Q$.

Suppose that $\left(\pi^{*}, \lambda_{1}^{*}, \lambda_{2}^{*}\right)$ attains the sup-sup-inf in Problem 1. Then, $X_{T}^{\pi^{*}}$ is expressed as (6) with $\lambda_{2}=\lambda_{2}^{*}$, since $\left(\pi^{*}, \lambda_{1}^{*}\right)$ is the saddle point for given $\lambda_{2}^{*} \in \tilde{\Lambda}_{2}$, and the portfolio process $\pi^{*}$ that generates $X_{T}^{\pi^{*}}$ at $T$ is given through the following expression on the discounted terminal wealth $\tilde{X}_{T}^{\pi^{*}}:=X_{T}^{\pi^{*}} \exp \left(-\int_{0}^{T} r_{s} d s\right)$. (See Section 3.1 in Ocone and Karatzas [15] for instance.)

$$
\tilde{X}_{T}^{\pi^{*}}=x+\int_{0}^{T} \psi_{t}^{\top} d B_{t}^{Q} .
$$

where $\psi$ is obtained by $\psi_{i, t}=E^{Q}\left[D_{i, t} \tilde{X}_{T}^{\pi^{*}}+\right.$ $\left.\tilde{X}_{T}^{\pi^{*}} \sum_{j=1}^{d} \int_{t}^{T} D_{i, t} \theta_{j, s} d B_{j, s}^{Q} \mid \mathcal{F}_{t}\right]$.

The portfolio process $\pi^{*}$ is related with $\mathcal{R}^{d}$-valued progressively process $\psi$ as $\frac{\pi_{t}^{* \top}}{S_{0, t}} \sigma_{\mathbf{t}}=\psi_{\mathbf{t}}^{\top}$, and given by $\pi_{t}^{* \top}=$ $S_{0, t} \psi_{t}^{\top} \sigma_{\mathbf{t}}{ }^{-1}$.

Particularly, $\psi$ has the following expression when $\tilde{X}_{T}^{\pi^{*}}$ is $\mathcal{F}_{T}^{Q}$-measurable, which is satisfied if $\theta$ and $\lambda^{*}$ are proportional to the same stochastic processes as in the cases in Section VI. When $\tilde{X}_{T}^{\pi^{*}} \in \mathbf{D}_{1,1}^{Q}$, by Clark-Ocone formula (e.g. Theorem 4.1 in Nunno et al. [6]), $\psi_{t}=E^{Q}\left[D_{t}^{Q} \tilde{X}_{T}^{\pi^{*}} \mid \mathcal{F}_{t}^{Q}\right]$.

Since we suppose $r \equiv 0$, we have $\pi_{t}^{* \top}=\psi_{t}^{\top} \sigma_{\mathbf{t}}{ }^{-1}$, and $\tilde{X}_{T}^{\pi^{*}}=X_{T}^{\pi^{*}}$. We can further calculate $\psi$ with $X_{T}^{\pi^{*}}$ given in (6) with $\lambda_{2}=\lambda_{2}^{*}$.

Proposition 1. Let $\lambda^{*}=\left(\lambda_{1}^{*}, \lambda_{2}^{*}\right)$. When $X_{T}^{\pi^{*}} \in \mathbf{D}_{1,1}^{Q}$ and $\lambda^{*}, \theta \in L_{1,1}^{a, Q}, \pi_{t}^{* \top}=S_{0, t} \psi_{t}^{\top} \sigma_{\mathbf{t}}{ }^{-1}$, where $\psi$ has the following expression if it is well-defined.

$$
\begin{gathered}
\psi_{i, t}=E^{Q}\left[-I^{\prime}\left(\frac{y H_{0, T}}{\eta_{T}^{\lambda_{1}^{*}, \lambda_{2}^{*}}}\right) \frac{y H_{0, T}}{\eta_{T}^{\lambda_{1}^{*}, \lambda_{2}^{*}}}\right. \\
\left(\left(\lambda_{i, t}^{*}-\theta_{i, t}\right)-\frac{1}{2} \int_{t}^{T} D_{i, t}^{Q}\left|\lambda_{s}^{*}-\theta_{s}\right|^{2} d s\right. \\
\left.\left.+\sum_{j=1}^{d} \int_{t}^{T} D_{i, t}^{Q}\left(\lambda_{j, s}^{*}-\theta_{j, s}\right) d B_{j, s}^{Q}\right) \mid \mathcal{F}_{t}^{Q}\right] .
\end{gathered}
$$

Corollary 2. In particular, when $\lambda^{*}$ and $\theta$ are deterministic processes,

$$
\psi_{i, t}=E^{Q}\left[-I^{\prime}\left(\frac{y H_{0, T}}{\eta_{T}^{\lambda_{1}^{*}, \lambda_{2}^{*}}}\right) \frac{y H_{0, T}}{\eta_{T}^{\lambda_{1}^{*}, \lambda_{2}^{*}}} \mid \mathcal{F}_{t}^{Q}\right]\left(\lambda_{i, t}^{*}-\theta_{i, t}\right) .
$$

Proof. By applying Clark-Ocone formula (e.g. Theorem 4.1 in Nunno et al. [6]) to $\tilde{X}_{T}^{\pi^{*}}$, we obtain the result. See Appendix B for details.

\section{EXPONENTIAL UTILITY CASES}

In this section, we present examples, in which the sup-supinf problem in Problem 1 in Section IV is explicitly solved and the optimal portfolio under conservative and aggressive sentiments in Section $\mathrm{V}$ is obtained, with an exponential utility where $u(x)=-\frac{1}{p} \exp (-p x), p>0$, and $I(y)=\left(u^{\prime}\right)^{-1}(y)=$ $-\frac{\log y}{p}$. For the cases with a $\log$ utility, see Appendix D in the full version of this paper [22].

Particularly, we present two cases where $\bar{\lambda}_{j}$, the bound defining $\Lambda_{j}, j=1,2$, and the market price of risk $-\theta$ are stochastic and investigate the impact of the conservative and aggressive sentiments on the optimal portfolio process.

\section{A. Brownian case}

Firstly, we consider a case where $\bar{\lambda}_{j}, j=1,2$, the processes defining the bound in $\Lambda_{j}$ and the market price of risk $-\theta$ are proportional to a Brownian motion $B^{\bar{\lambda}_{1}, \bar{\lambda}_{2}}$ under the probability measure $P^{\bar{\lambda}_{1}, \bar{\lambda}_{2}}$.

Example 1. (Brownian case)

Let $\bar{\sigma}_{\lambda, j}, \sigma_{\theta, j}, j=1,2$, be constants satisfying $\bar{\sigma}_{\lambda, j}-\sigma_{\theta, j}>$ $0,-\sigma_{\theta, j}>0, j=1,2$ with $\bar{\sigma}_{\lambda, 1} \leq 0, \bar{\sigma}_{\lambda, 2} \geq 0$.

Let

$$
\begin{aligned}
& \bar{\lambda}_{j, t}=\bar{\sigma}_{\lambda, j} \int_{0}^{t} e^{-\bar{\sigma}_{\lambda, j}(t-s)} d B_{j, s}, \\
& \theta_{j, t}=\sigma_{\theta, j} \int_{0}^{t} e^{-\bar{\sigma}_{\lambda, j}(t-s)} d B_{j, s} .
\end{aligned}
$$

In this case, $\bar{\lambda}$ and $\theta$ are rewritten as $\bar{\lambda}_{j, t}=\bar{\sigma}_{\lambda, j} B_{j, t}^{\bar{\lambda}_{1}, \bar{\lambda}_{2}}$ and $\theta_{j, t}=\sigma_{\theta, j} B_{j, t}^{\bar{\lambda}_{1}, \bar{\lambda}_{2}}$, since $B_{j, t}^{\bar{\lambda}_{1}, \bar{\lambda}_{2}}=\int_{0}^{t} e^{-\bar{\sigma}_{\lambda, j}(t-s)} d B_{j, s}$ is an Ornstein-Uhlenbeck process that satisfies $B_{j, t}^{\bar{\lambda}_{1}, \bar{\lambda}_{2}}=B_{j, t}-$ $\bar{\sigma}_{\lambda, j} \int_{0}^{t} B_{j, s}^{\bar{\lambda}_{1}, \bar{\lambda}_{2}} d s$.

Let $\Lambda_{1}$ and $\tilde{\Lambda}_{2}$ be a set of $\lambda_{1}$ and $\lambda_{2}$, respectively, satisfying $\lambda_{j, t}=\sigma_{\lambda, j} \int_{0}^{t} e^{-\bar{\sigma}_{\lambda, j}(t-s)} d B_{j, s},=\sigma_{\lambda, j} B_{j, t}^{\bar{\lambda}_{1}, \bar{\lambda}_{2}}, j=1,2$, where $\sigma_{\lambda, j}, j=1,2$, are constants satisfying $-\left|\bar{\sigma}_{\lambda, j}\right| \leq$ $\sigma_{\lambda, j} \leq\left|\bar{\sigma}_{\lambda, j}\right|$.

Then, $\lambda_{j, t}^{*}=\bar{\lambda}_{j, t}, j=1,2$, and $\pi^{*}$ generating the terminal wealth $X_{T}^{\pi^{*}}=-\frac{1}{p} \log \left(\frac{y H_{0, T}}{\eta_{T}^{\lambda_{1}, \lambda_{2}}}\right)$ where $y=\exp (-p x-$ $\left.E^{Q}\left[\log H_{0, T}-\log \eta_{T}^{\bar{\lambda}_{1}, \bar{\lambda}_{2}}\right]\right)$ attain the sup-sup-inf in Problem 1. This is confirmed as follows. First, (6) and (8) in (i),(ii) in Section IV are satisfied, and thus $\lambda_{1}^{*}=\bar{\lambda}_{1}$ and $\pi^{*}$ generating $X_{T}^{\pi^{*}}=-\frac{1}{p} \log \left(\frac{y H_{0, T}}{\eta_{T}^{\lambda_{1}, \lambda_{2}}}\right)$ provide a saddle point. Then by considering the maximization with respect to $\lambda_{2}$, we obtain the result. For details, see Appendix C.

Remark 3. Since $\bar{\lambda}_{1}=\bar{\sigma}_{\lambda, 1} B_{1}^{\bar{\lambda}_{1}, \bar{\lambda}_{2}}, \lambda_{2}=\sigma_{\lambda, 2} B_{2}^{\bar{\lambda}_{1}, \bar{\lambda}_{2}}$ and $\theta_{j}=\sigma_{\theta, j} B_{j}^{\bar{\lambda}_{1}, \bar{\lambda}_{2}}, j=1,2$, and $B_{j}^{\bar{\lambda}_{1}, \bar{\lambda}_{2}}$ is an OrnsteinUhlenbeck process of $B_{j}^{\bar{\lambda}_{1}, \lambda_{2}}, B_{1, t}^{\bar{\lambda}_{1}, \bar{\lambda}_{2}}=B_{1, t}^{\bar{\lambda}_{1}, \lambda_{2}}, B_{2, t}^{\bar{\lambda}_{1}, \bar{\lambda}_{2}}=$ $\int_{0}^{t} e^{-\left(\bar{\sigma}_{\lambda, 2}-\sigma_{\lambda, 2}\right)(t-s)} d B_{2, s}^{\bar{\lambda}_{1}, \lambda_{2}}$, thus $X_{T}^{\pi^{*}}=-\frac{1}{p} \log \left(\frac{y H_{0, T}}{\eta_{T}^{\lambda_{1}, \lambda_{2}}}\right)$ is $\mathcal{F}_{T}^{\bar{\lambda}_{1}, \lambda_{2}}$-measurable and in particular in $\mathbf{D}_{1,1}^{\bar{\lambda}_{1}, \lambda_{2}}$. Thus, we can apply Corollary 1 and confirm that $\lambda_{1}^{*}\left(\lambda_{2}\right)=\bar{\lambda}_{2}$ and $\pi^{*}\left(\lambda_{2}\right)$ generating $X_{T}^{\pi^{*}}=-\frac{1}{p} \log \left(\frac{y H_{0, T}}{\eta_{T}^{\lambda_{1}, \lambda_{2}}}\right)$ provide a saddle point for given $\lambda_{2} \in \tilde{\Lambda}_{2}$. Then, it follows that $\lambda_{2}^{*}=\bar{\lambda}_{2}$ attains $\sup _{\lambda_{2} \in \tilde{\Lambda}_{2}} J\left(\pi^{*}\left(\lambda_{2}\right), \lambda_{1}^{*}\left(\lambda_{2}\right), \lambda_{2}\right)$.

Moreover, for the calculation of the optimal portfolio $\pi^{*}$, since $\bar{\lambda}_{j}=\bar{\sigma}_{\lambda, j} B_{j}^{\bar{\lambda}_{1}, \bar{\lambda}_{2}}$, and $\theta_{j}=\sigma_{\theta, j} B_{j}^{\lambda_{1}, \bar{\lambda}_{2}}, j=1,2$, and $B_{j}^{\bar{\lambda}_{1}, \bar{\lambda}_{2}}$ is an Ornstein-Uhlenbeck process of $B_{j}^{Q}, B_{j, t}^{\bar{\lambda}_{1}, \bar{\lambda}_{2}}=$ 
$\int_{0}^{t} e^{-\left(\bar{\sigma}_{\lambda, j}-\sigma_{\theta, j}\right)(t-s)} d B_{j, s}^{Q}$, thus $X_{T}^{\pi^{*}}=-\frac{1}{p} \log \left(\frac{y H_{0, T}}{\eta_{T}^{\lambda_{1}, \lambda_{2}}}\right)$ is $\mathcal{F}_{T}^{Q}$-measurable and in particular in $\mathbf{D}_{1,1}^{Q}$. Thus, we can apply Proposition 1, and the optimal portfolio $\pi^{*}$ is calculated as $\pi_{t}^{* \top}=\psi_{t}^{\top} \sigma_{\mathbf{t}}^{-1}$ with

$$
\psi_{j, t}=\frac{1}{2 p}\left(1+e^{-2\left(\bar{\sigma}_{\lambda, j}-\sigma_{\theta, j}\right)(T-t)}\right)\left(\bar{\sigma}_{\lambda, j}-\sigma_{\theta, j}\right) B_{j, t}^{\bar{\lambda}_{1}, \bar{\lambda}_{2}} .
$$

For details, see Appendix C.1 in the full version of this paper [22].

Remark 4. With this $\bar{\lambda}$ in (17), the first integrability condition in (1) is not satisfied. We can restrict $\bar{\lambda}$ so that it satisfies the integrability condition. Specifically, we can use $f\left(B^{\bar{\lambda}_{1}, \bar{\lambda}_{2}}\right)$ instead of $B^{\bar{\lambda}_{1}, \bar{\lambda}_{2}}$ with $f: \mathcal{R} \rightarrow \mathcal{R}$ such that $f$ is smooth, increasing, $f(x)=x,|x| \leq R$ for some $R>0$, and $\lim _{x \rightarrow+-\infty} \frac{|f(x)|}{\log |x|}<C, C>0$. The same thing holds for the square-root case in (20).

\section{B. Impact of the sentiments on the position in the Brownian} case

In this subsection, we investigate the impact of the conservative and aggressive sentiments $\lambda_{j}^{*}$ on the position on the Brownian component $\psi_{j}, j=1,2$ in the Brownian case with the exponential utility. We note that $\psi_{j}$ is interpreted as the position on the Brownian component $B_{j}, j=1,2$, as $\psi_{t}=\pi_{t}^{*} \sigma_{t}$ and (16) indicate.

First of all, we consider the base case where $\bar{\sigma}_{\lambda, j}=$ $0, j=1,2$. The expression of $\psi_{j}$ in (18) indicates that when $B_{j, t}^{\bar{\lambda}_{1}, \bar{\lambda}_{2}}>0(<0), j=1,2$, the position $\psi_{j, t}$ on the $j$-th Brownian component is positive (negative), which means long (short).

Next, when $\bar{\sigma}_{\lambda, 1}<0, \bar{\sigma}_{\lambda, 2}>0$, since $\bar{\sigma}_{\lambda, j}-\sigma_{\theta, j}>0, \psi_{j, t}$ in (18) has the same sign as $B_{j, t}^{\bar{\lambda}_{1}, \bar{\lambda}_{2}}$ as in the base case. Then, we observe the following for the expression of $\psi_{j}$ in (18).

- When $B_{1, t}^{\bar{\lambda}_{1}, \bar{\lambda}_{2}}>0(<0), \lambda_{1, t}^{*}=\bar{\sigma}_{\lambda, 1} B_{1, t}^{\bar{\lambda}_{1}, \bar{\lambda}_{2}}$ with $\bar{\sigma}_{\lambda, 1}<0$ implies a negative (positive) bias $\lambda_{1, t}^{*}<0(>0)$ for $B_{1, t}$. Since $\psi_{1, t}$, the position on the first Brownian component $B_{1}$, is long (short), which is compatible with the role of $\lambda_{1}^{*}$ as the conservative sentiment. Similarly, $\bar{\sigma}_{\lambda, 2}>0$ indicates a positive (negative) bias on $B_{2, t}$ when $B_{2, t}^{\bar{\lambda}_{1}, \bar{\lambda}_{2}}>0(<0)$, which implies that $\lambda_{2}^{*}$ is viewed as the aggressive sentiment.

- Compared with the base case where $\bar{\sigma}_{\lambda, 1}=0, \lambda_{1}^{*}=$ $\bar{\sigma}_{\lambda, 1} B_{1, t}^{\bar{\lambda}_{1}, \bar{\lambda}_{2}}$ with $\bar{\sigma}_{\lambda, 1}<0$ and $B_{1, t}^{\bar{\lambda}_{1}, \bar{\lambda}_{2}}>0(<0)$ indicates that the position on the first Brownian component $\psi_{1, t}$ is smaller (the short amount is smaller) when the position is long (short). This agrees with the fact that the investor maximizes the expected utility under the conservative sentiment. Similar arguments hold for the effect of the aggressive sentiment $\lambda_{2}^{*}$.

Furthermore, we separate the expression of $\psi_{j, t}$ into the first and the second term as $\psi_{j, t}=\frac{1}{p}\left(\left(\bar{\sigma}_{\lambda, j}-\sigma_{\theta, j}\right) B_{j, t}^{\bar{\lambda}_{1}, \bar{\lambda}_{2}}-\frac{1}{2}(1-\right.$ $\left.\left.e^{-2\left(\bar{\sigma}_{\lambda, j}-\sigma_{\theta, j}\right)(T-t)}\right)\left(\bar{\sigma}_{\lambda, j}-\sigma_{\theta, j}\right) B_{j, t}^{\bar{\lambda}_{1}, \bar{\lambda}_{2}}\right)$. This indicates that the first term contains $\lambda_{j, t}^{*}-\theta_{j, t}$ the sentiment added market price of risk, and the second term offsets the first term while it vanishes as time approaches maturity. Then, the effect of $\bar{\sigma}_{\lambda, 1}<0\left(\bar{\sigma}_{\lambda, 2}>0\right)$ on the first term is the same as we discussed on the whole $\psi_{j, t}$ above.

The effect of $\bar{\sigma}_{\lambda, 1}<0\left(\bar{\sigma}_{\lambda, 2}>0\right)$ on the second term is as follows. We set $g\left(\bar{\sigma}_{\lambda, j}\right)=-\frac{1}{2}\left(1-e^{-2\left(\bar{\sigma}_{\lambda, j}-\sigma_{\theta, j}\right)(T-t)}\right)\left(\bar{\sigma}_{\lambda, j}-\right.$ $\left.\sigma_{\theta, j}\right)$. Then, it follows that $g^{\prime}\left(\bar{\sigma}_{\lambda, j}\right)<0$.

This indicates that compared with the base case where $\bar{\sigma}_{\lambda, j}=0, \bar{\sigma}_{\lambda, 1}<0\left(\bar{\sigma}_{\lambda, 2}>0\right)$ makes the position $\psi_{1}\left(\psi_{2}\right)$ larger (the short amount is larger) and mitigates the first term effect.

\section{Square-root case}

Finally, we consider the case in which $\bar{\lambda}_{j}, j=1,2$, defining the bound in $\Lambda_{j}$ and the market price of risk $-\theta$ are proportional to a square-root of a square-root process $V$.

Example 2. (Square-root case)

Let $\bar{\sigma}_{\lambda, j}, \sigma_{\theta, j}, j=1,2$, be constants satisfying $\bar{\sigma}_{\lambda, j}-\sigma_{\theta, j}>$ $0,-\sigma_{\theta, j}>0, j=1,2$ with $\bar{\sigma}_{\lambda, 1} \leq 0, \bar{\sigma}_{\lambda, 2} \geq 0$, and $\sigma_{v, j}>$ $0, j=1,2$.

Let $V$ be a square-root process satisfying a SDE

$$
\begin{gathered}
d V_{j, t} \\
=\left(\kappa_{j}\left(\bar{\theta}_{j}-V_{j, t}\right)-\sigma_{v, j} \bar{\sigma}_{\lambda, j} V_{j, t}\right) d t+\sigma_{v, j} \sqrt{V_{j, t}} d B_{j, t} \\
V_{j, 0}>0, j=1,2
\end{gathered}
$$

where $\kappa_{j}, \bar{\theta}_{j}$ are positive constants satisfying $2 \kappa_{j} \bar{\theta}_{j} \geq \sigma_{v, j}^{2}$.

Also, let

$$
\bar{\lambda}_{j, s}=\bar{\sigma}_{\lambda, j} \sqrt{V_{j, s}}, \theta_{j, s}=\sigma_{\theta, j} \sqrt{V_{j, s}} .
$$

Let $\Lambda_{1}$ and $\tilde{\Lambda}_{2}$ be a set of $\lambda_{1}$ and $\lambda_{2}$ of the form $\lambda_{j, s}=$ $\sigma_{\lambda, j} \sqrt{V_{j, s}}$, respectively, where $\sigma_{\lambda, j}$ is a constant satisfying $-\left|\bar{\sigma}_{\lambda, j}\right| \leq \sigma_{\lambda, j} \leq\left|\bar{\sigma}_{\lambda, j}\right|$.

Then, $\lambda_{j}^{*}, j=1,2$ defined as $\lambda_{j, s}^{*}=\bar{\sigma}_{\lambda, j} \sqrt{V_{j, s}}$ and $\pi^{*}$ generating the terminal wealth $X_{T}^{\pi^{*}}=-\frac{1}{p} \log \left(\frac{y H_{0, T}}{\eta_{T}^{\lambda_{1}, \lambda_{2}}}\right)$ where $y=\exp \left(-p x-E^{Q}\left[\log H_{0, T}-\log \eta_{T}^{\bar{\lambda}_{1}, \bar{\lambda}_{2}}\right]\right)$ attain the sup-sup-inf in Problem 1. This is confirmed as follows. First, (6) and (8) in (i),(ii) in Section IV are satisfied, and thus $\lambda_{1}^{*}=\bar{\lambda}_{1}$ and $\pi^{*}$ generating $X_{T}^{\pi^{*}}=-\frac{1}{p} \log \left(\frac{y H_{0, T}}{\eta_{T}^{\lambda_{1}, \lambda_{2}}}\right)$ provide a saddle point. By considering the maximization on $\lambda_{2}$, we obtain the result. For details, see Appendix D.

Remark 5. Since $\bar{\lambda}_{1}=\bar{\sigma}_{\lambda, 1} \sqrt{V_{1}}, \lambda_{2}=\sigma_{\lambda, 2} \sqrt{V_{2}}$ and $\theta_{j}=\sigma_{\theta, j} \sqrt{V_{j}}, j=1,2$, and $V_{j}$ is a square-root process, which is expressed as a unique strong solution of SDE $d V_{1, t}=\kappa_{1}\left(\bar{\theta}_{1}-V_{1, t}\right) d t+\sigma_{v, 1} \sqrt{V_{1, t}} d B_{1, t}^{\bar{\lambda}_{1}, \lambda_{2}}, d V_{2, t}=\left(\kappa_{2}\left(\bar{\theta}_{2}-\right.\right.$ $\left.\left.V_{2, t}\right)-\sigma_{v, 2}\left(\bar{\sigma}_{\lambda, 2}-\sigma_{\lambda, 2}\right) V_{2, t}\right) d t+\sigma_{v, 2} \sqrt{V_{2, t}} d B_{2, t}^{\bar{\lambda}_{1}, \lambda_{2}}, V_{j, 0}>$ $0, j=1,2$, thus $X_{T}^{\pi^{*}}=-\frac{1}{p} \log \left(\frac{y H_{0, T}}{\eta_{T}^{\lambda_{1}, \lambda_{2}}}\right)$ is $\mathcal{F}_{T}^{\bar{\lambda}_{1}, \lambda_{2}}$. measurable and in particular in $\mathbf{D}_{1,1}^{\bar{\lambda}_{1}, \lambda_{2}}$. Thus, we can apply Corollary 1 and confirm that $\pi^{*}\left(\lambda_{2}\right)$ generating $X_{T}^{\pi^{*}}=$ 
$-\frac{1}{p} \log \left(\frac{y H_{0, T}}{\eta_{T}^{\lambda_{1}, \lambda_{2}}}\right)$ and $\lambda_{1}^{*}\left(\lambda_{2}\right)=\bar{\lambda}_{1}$ provide a saddle point for given $\lambda_{2} \in \tilde{\Lambda}_{2}$. Then, it follows that $\lambda_{2}^{*}=\bar{\lambda}_{2}$ attains $\sup _{\lambda_{2} \in \tilde{\Lambda}_{2}} J\left(\pi^{*}\left(\lambda_{2}\right), \lambda_{1}^{*}\left(\lambda_{2}\right), \lambda_{2}\right)$.

Moreover, for the calculation of the optimal portfolio, since $\bar{\lambda}_{1}=\bar{\sigma}_{\lambda, 1} \sqrt{V_{1}}, \lambda_{2}=\sigma_{\lambda, 2} \sqrt{V_{2}}$ and $\theta_{j}=\sigma_{\theta, j} \sqrt{V_{j}}, j=1,2$, and $V_{j}$ is a square-root process, which is expressed as a unique strong solution of $\operatorname{SDE} d V_{j, t}=\left(\kappa_{j}\left(\bar{\theta}_{j}-V_{j, t}\right)-\sigma_{v, j}\left(\bar{\sigma}_{\lambda, j}-\right.\right.$ $\left.\left.\sigma_{\theta, j}\right) V_{j, t}\right) d t+\sigma_{v, j} \sqrt{V_{j, t}} d B_{j, t}^{Q}, \quad V_{j, 0}>0, j=1,2$, thus $X_{T}^{\pi^{*}}=-\frac{1}{p} \log \left(\frac{y H_{0, T}}{\eta_{T}^{\lambda_{1}, \lambda_{2}}}\right)$ is $\mathcal{F}_{T}^{Q}$-measurable and in particular in $\mathbf{D}_{1,1}^{Q}$. Thus, we can apply Proposition 1 and calculate the optimal portfolio process $\pi^{*}$ as $\pi^{* \top}=\psi_{t}^{\top} \sigma_{\mathbf{t}}^{-1}$ with

$$
\begin{aligned}
\psi_{j, t}= & \frac{1}{p}\left[\left(\bar{\sigma}_{\lambda, j}-\sigma_{\theta, j}\right) \sqrt{V_{j, t}}\right. \\
& \left.-\left(\bar{\sigma}_{\lambda, j}-\sigma_{\theta, j}\right)^{2} E^{Q}\left[\int_{t}^{T} \sqrt{V_{j, s}} D_{j, s}^{Q} \sqrt{V_{j, s}} d s \mid \mathcal{F}_{t}^{Q}\right]\right],
\end{aligned}
$$

where

$$
\begin{gathered}
d V_{j, t}= \\
\left(\kappa_{j}\left(\bar{\theta}_{j}-V_{j, t}\right)+\sigma_{v, j}\left(\sigma_{\theta, j}-\bar{\sigma}_{\lambda, j}\right) V_{j, t}\right) d t+\sigma_{v, j} \sqrt{V_{j, t}} d B_{j, t}^{Q},
\end{gathered}
$$

and

$$
\begin{gathered}
D_{j, t}^{Q} \sqrt{V_{j, s}}=\frac{\sigma_{v, j}}{2} \times \\
\exp \left(\int_{t}^{s}\left[-\frac{\kappa_{j}+\sigma_{v, j}\left(\bar{\sigma}_{\lambda, j}-\sigma_{\theta, j}\right)}{2}-\frac{\frac{\kappa_{j} \bar{\theta}_{j}}{2}-\frac{\sigma_{v, j}^{2}}{8}}{V_{j, u}}\right] d u\right) .
\end{gathered}
$$

Here, we note that $E^{Q}\left[\int_{t}^{T} \sqrt{V_{j, s}} D_{j, s}^{Q} \sqrt{V_{j, s}} d s \mid \mathcal{F}_{t}^{Q}\right]<$ $\infty$ in (21), since $-\frac{\kappa_{j}+\sigma_{v, j}\left(\bar{\sigma}_{\lambda, j}-\sigma_{\theta, j}\right)}{2}-\frac{\frac{\kappa_{j} \bar{\theta}_{j}}{2}-\frac{\sigma_{v, j}^{2}}{8}}{V_{j, u}}<0$, and thus $\left|D_{j, t}^{Q} \sqrt{V_{j, s}}\right| \leq \frac{\sigma_{v, j}}{2}$, also $E^{Q}\left[\int_{0}^{T} \sqrt{V_{j, s}} d s\right] \leq$ $\sqrt{T} \sqrt{E^{Q}\left[\int_{0}^{T} V_{j, s} d s\right]}<\infty$, and thus $E^{Q}\left[\int_{0}^{T} \sqrt{V_{j, s}} D_{j, t}^{Q} \sqrt{V_{j, s}} d s\right]<\infty$.

For details, see Appendix C.2 in the full version of this paper [22].

\section{Impact of the sentiments on the position in the square-root case}

Firstly, for the first term in (21), we observe the same effect of the conservative and aggressive sentiments $\lambda_{j}^{*}, j=1,2$ on the positions of the Brownian components $\psi_{j}$, as in the Brownian case. That is, since $\sigma_{\lambda, j}-\sigma_{\theta, j}>0, j=1,2$, the position on the Brownian component $B_{1}\left(B_{2}\right)$ is long, while $\sigma_{\lambda, 1}<0\left(\sigma_{\lambda, 2}>0\right)$ implies a negative (positive) bias on $B_{1}$ $\left(B_{2}\right)$. Moreover, compared with the base case where $\bar{\sigma}_{j}=$ $0, j=1,2, \sigma_{\lambda, 1}<0\left(\sigma_{\lambda, 2}>0\right)$ makes the position smaller (larger).

Secondly, for the second term in (21), $\bar{\sigma}_{\lambda, 1}<0\left(\bar{\sigma}_{\lambda, 2}>0\right)$ makes the position larger (smaller), which mitigates the effect in the first term. We note that we can calculate $\psi_{j, t}$ numerically by simulating $E^{Q}\left[\int_{t}^{T} \sqrt{V_{j, s}} D_{j, s}^{Q} \sqrt{V_{j, s}} d s \mid \mathcal{F}_{t}^{Q}\right]$ in (21) by (22) with (23).

\section{CONCLUSION}

In this work, we have proposed a portfolio optimization with a choice of a probability measure and presented its application to an optimal investment problem under conservative and aggressive sentiments. Firstly, we have formulated this new problem as a combination of the optimal portfolio problem and the sup-inf problem on a choice of a probability measure. Then we have provided the conditions under which the supsup-inf problem is solved by a Malliavin calculus approach. Finally, we have obtained the optimal portfolio expression and investigated the effects of the sentiments on the portfolio for the exponential utility cases. Developing a text mining approach with machine learning to estimate and predict market sentiment factors as in Nakatani et al. [13] and applying machine learning to numerical solutions of relevant highdimensional BSDEs are our main future research topics.

\section{REFERENCES}

[1] Alos, E., \& Ewald, C. O. (2008). Malliavin differentiability of the Heston volatility and applications to option pricing. Advances in Applied Probability, 40(1), 144-162.

[2] Benavoli, A., \& Chisci, L. (2011). Robust stochastic control based on imprecise probabilities. IFAC Proceedings Volumes, 44(1), 4606-4613.

[3] Bensoussan, A., Siu, C. C., Yam, S. C. P., \& Yang, H. (2014). A class of non-zero-sum stochastic differential investment and reinsurance games. Automatica, 50(8), 2025-2037.

[4] Calafiore, G. C. (2013). Direct data-driven portfolio optimization with guaranteed shortfall probability. Automatica, 49(2), 370-380.

[5] Calafiore, G. C. (2008). Multi-period portfolio optimization with linear control policies. Automatica, 44(10), 2463-2473.

[6] Di Nunno, G., Oksendal, B. K., \& Proske, F. (2009). Malliavin calculus for Levy processes with applications to finance (Vol. 2). Berlin: Springer.

[7] Dombrovskii, V., Obyedko, T., \& Samorodova, M. (2018). Model predictive control of constrained Markovian jump nonlinear stochastic systems and portfolio optimization under market frictions. Automatica, 87, 61-68.

[8] S. E., Karatzas, I., \& Shreve,(1998). Methods of mathematical finance (Vol. 39, pp. xvi+-407). New York: Springer.

[9] Karatzas, I., \& Shreve, S. (2012). Brownian motion and stochastic calculus (Vol. 113). Springer Science \& Business Media.

[10] Li, N., Wang, G., \& Wu, Z. (2020). Linear-quadratic optimal control for time-delay stochastic system with recursive utility under full and partial information. Automatica, 121, 109169.

[11] Ma, G., Siu, C. C., Zhu, S. P., \& Elliott, R. J. (2020). Optimal portfolio execution problem with stochastic price impact. Automatica, 112, 108739 .

[12] Mukuddem-Petersen, J., \& Petersen, M. A. (2006). Bank management via stochastic optimal control. Automatica, 42(8), 1395-1406.

[13] Nakatani, S., Nishimura, K. G., Saito, T., \& Takahashi, A. (2020). Interest rate model with investor attitude and text mining. IEEE Access, $8,86870-86885$.

[14] Nishimura, K. G., Sato, S., \& Takahashi, A. (2019). Term Structure Models During the Global Financial Crisis: A Parsimonious Text Mining Approach. Asia Pacific Financial Markets, https://doi.org/10.1007/s10690-018-09267-9.

[15] Ocone, D. L., \& Karatzas, I. (1991). A generalized Clark representation formula, with application to optimal portfolios. Stochastics: An International Journal of Probability and Stochastic Processes, 34(3-4), 187-220.

[16] Pardoux, E., \& Rascanu, A. (2014). Stochastic Differential Equations. In Stochastic Differential Equations, Backward SDEs, Partial Differential Equations (pp. 135-227). Springer, Cham.

[17] Petersen, I. R., James, M. R., \& Dupuis, P. (2000). Minimax optimal control of stochastic uncertain systems with relative entropy constraints. IEEE Transactions on Automatic Control, 45(3), 398-412. 
[18] Pun, C. S. (2018). Robust time-inconsistent stochastic control problems. Automatica, 94, 249-257.

[19] Saito, T., Takahashi, A.(2022). Sup-inf/inf-sup problem on choice of a probability measure by FBSDE approach. IEEE Transactions on Automatic Control, DOI 10.1109/TAC.2021.3058422.

[20] Saito, T., \& Takahashi, A. (2017). Derivatives pricing with market impact and limit order book. Automatica, 86, 154-165.

[21] Saito, T., \& Takahashi, A. (2019). Stochastic differential game in high frequency market. Automatica, 104, 111-125.

[22] Saito, T., \& Takahashi, A. (2021). Portfolio optimization with choice of a probability measure (full version). SSRN, http://ssrn.com/abstract=3816173.

[23] Takahashi, A., \& Yoshida, N. (2004). An asymptotic expansion scheme for optimal investment problems. Statistical Inference for Stochastic Processes, 7(2), 153-188.

[24] Wang, G., \& Yu, Z. (2012). A partial information non-zero sum differential game of backward stochastic differential equations with applications. Automatica, 48(2), 342-352.

[25] Yan, T., \& Wong, H. Y. (2019). Open-loop equilibrium strategy for mean variance portfolio problem under stochastic volatility. Automatica, 107, 211-223.

[26] Yiu, K. F. C., Liu, J., Siu, T. K., \& Ching, W. K. (2010). Optimal portfolios with regime switching and value-at-risk constraint. Automatica, 46(6), 979-989.

\section{APPENDIX}

\section{A. Theorem 1 and Corollary 1}

In the following, we calculate $Z_{t}^{\pi^{*}, \lambda_{1}^{*}, \lambda_{2}}$ for a general stochastic $r \in$ $L_{1,1}^{a}$ case.

By Theorem 2.5 in Ocone and Karatzas [15], we have

$$
\begin{gathered}
Z_{t}^{\pi^{*}, \lambda_{1}^{*}, \lambda_{2}} \\
=E^{P^{\lambda_{1}^{*}, \lambda_{2}}}\left[D_{t} u\left(X_{T}^{\pi^{*}}\right)+u\left(X_{T}^{\pi^{*}}\right) \int_{t}^{T} D_{t} \tilde{\lambda}_{s} d B_{s}^{\lambda_{1}^{*}, \lambda_{2}} \mid \mathcal{F}_{t}\right] .
\end{gathered}
$$

Since

$$
u\left(X_{T}^{\pi^{*}}\right)=u\left(I\left(\frac{y H_{0, T}}{\eta_{T}^{\lambda_{1}^{*}, \lambda_{2}}}\right)\right),
$$

we have

$$
\begin{aligned}
& D_{t} u\left(X_{T}^{\pi^{*}}\right)=u^{\prime}\left(I\left(\frac{y H_{0, T}}{\eta_{T}^{\lambda_{1}^{*}, \lambda_{2}}}\right)\right) D_{t}\left(I\left(\frac{y H_{0, T}}{\eta_{T}^{\lambda_{1}^{*}, \lambda_{2}}}\right)\right) \\
= & \left(\frac{y H_{0, T}}{\eta_{T}^{\lambda_{1}^{*}, \lambda_{2}}}\right) I^{\prime}\left(\frac{y H_{0, T}}{\eta_{T}^{\lambda_{1}^{*}, \lambda_{2}}}\right) D_{t}\left(\exp \left(\log \frac{y H_{0, T}}{\eta_{T}^{\lambda_{1}^{*}, \lambda_{2}}}\right)\right) \\
= & I^{\prime}\left(\frac{y H_{0, T}}{\eta_{T}^{\lambda_{1}^{*}, \lambda_{2}}}\right)\left(\frac{y H_{0, T}}{\eta_{T}^{\lambda_{1}^{*}, \lambda_{2}}}\right)^{2} D_{t}\left(\log H_{0, T}-\log \eta_{T}^{\lambda_{1}^{*}, \lambda_{2}}\right) .
\end{aligned}
$$

We rewrite $\log H_{0, T}$ and $\log \eta_{T}^{\lambda_{1}^{*}, \lambda_{2}}$ as

$$
\begin{aligned}
\log H_{0, T}= & -\int_{0}^{T} r_{s} d s+\int_{0}^{T} \theta_{s}^{\top} d B_{s}-\frac{1}{2} \int_{0}^{T}\left|\theta_{s}\right|^{2} d s \\
= & -\int_{0}^{T} r_{s} d s+\int_{0}^{T} \theta_{s}^{\top} d B_{s}^{\lambda_{1}^{*}, \lambda_{2}}-\frac{1}{2} \int_{0}^{T}\left|\theta_{s}\right|^{2} d s \\
& +\int_{0}^{T} \theta_{s}^{\top} \tilde{\lambda}_{s} d s,
\end{aligned}
$$

and

$$
\begin{aligned}
\log \eta_{T}^{\lambda_{1}^{*}, \lambda_{2}} & =\int_{0}^{T} \tilde{\lambda}_{s}^{\top} d B_{s}-\frac{1}{2} \int_{0}^{T}\left|\tilde{\lambda}_{s}\right|^{2} d s \\
& =\int_{0}^{T} \tilde{\lambda}_{s}^{\top} d B_{s}^{\lambda_{1}^{*}, \lambda_{2}}+\frac{1}{2} \int_{0}^{T}\left|\tilde{\lambda}_{s}\right|^{2} d s
\end{aligned}
$$

where

$$
d B_{u}=d B_{s}^{\lambda_{1}^{*}, \lambda_{2}}+\tilde{\lambda}_{s} d s .
$$

Thus, we have

$$
\begin{aligned}
D_{t}\left(\log H_{0, T}\right)= & -\int_{t}^{T} D_{t} r_{s} d s+\theta_{t}+\int_{t}^{T} D_{t} \theta_{s} d B_{s} \\
& -\int_{t}^{T}\left(D_{t} \theta_{s}\right) \theta_{s} d s \\
= & -\int_{t}^{T} D_{t} r_{s} d s+\theta_{t}+\int_{t}^{T} D_{t} \theta_{s} d B_{s}^{\lambda_{1}^{*}, \lambda_{2}} \\
& +\int_{t}^{T}\left(D_{t} \theta_{s}\right)\left(\tilde{\lambda}_{s}-\theta_{s}\right) d s,
\end{aligned}
$$

and

$$
\begin{aligned}
D_{t}\left(\log \eta_{T}\right) & =\tilde{\lambda}_{t}+\int_{t}^{T} D_{t} \tilde{\lambda}_{s} d B_{s}-\int_{t}^{T}\left(D_{t} \tilde{\lambda}_{s}\right) \tilde{\lambda}_{s} d s \\
& =\tilde{\lambda}_{t}+\int_{t}^{T} D_{t} \tilde{\lambda}_{s} d B_{s}^{\lambda_{1}^{*}, \lambda_{2}} .
\end{aligned}
$$

Therefore,

$$
\begin{gathered}
Z_{t}^{\pi^{*}, \lambda_{1}^{*}, \lambda_{2}}=E^{P^{\lambda_{1}^{*}, \lambda_{2}}}\left[I^{\prime}\left(\frac{y H_{0, T}}{\eta_{T}^{\lambda_{1}^{*}, \lambda_{2}}}\right)\left(\frac{y H_{0, T}}{\eta_{T}^{\lambda_{1}^{*}, \lambda_{2}}}\right)^{2}\right. \\
\left(-\int_{t}^{T} D_{t} r_{s} d s-\left(\tilde{\lambda}_{t}-\theta_{t}\right)+\int_{t}^{T}\left(D_{t} \theta_{s}\right)\left(\tilde{\lambda}_{s}-\theta_{s}\right) d s\right. \\
\left.\left.-\int_{t}^{T} D_{t}\left(\tilde{\lambda}_{s}-\theta_{s}\right) d B_{s}^{\lambda_{1}^{*}, \lambda_{2}}\right)+u\left(X_{T}^{\pi^{*}}\right) \int_{t}^{T} D_{t} \tilde{\lambda}_{s} d B_{s}^{\lambda_{1}^{*}, \lambda_{2}} \mid \mathcal{F}_{t}\right] .
\end{gathered}
$$

In the case where $X_{T}^{\pi^{*}} \in \mathbf{D}_{1,1}^{\lambda_{1}^{*}, \lambda_{2}}$, by Clark-Ocone formula (e.g. Theorem 4.1 in Nunno et al. [6] ), we have

$$
Z_{t}^{\pi^{*}, \lambda_{1}^{*}, \lambda_{2}}=E^{P^{\lambda_{1}^{*}, \lambda_{2}}}\left[D_{t}^{\lambda_{1}^{*}, \lambda_{2}} u\left(X_{T}^{\pi^{*}}\right) \mid \mathcal{F}_{t}^{\lambda_{1}^{*}, \lambda_{2}}\right] .
$$

Then, the following calculation holds

$$
\begin{aligned}
& D_{t}^{\lambda_{1}^{*}, \lambda_{2}} u\left(X_{T}^{\pi^{*}}\right) \\
& =I^{\prime}\left(\frac{y H_{0, T}}{\eta_{T}^{\lambda_{1}^{*}, \lambda_{2}}}\right)\left(\frac{y H_{0, T}}{\eta_{T}^{\lambda_{1}^{*}, \lambda_{2}}}\right)^{2} D_{t}^{\lambda_{1}^{*}, \lambda_{2}}\left(\log H_{0, T}-\log \eta_{T}^{\lambda_{1}^{*}, \lambda_{2}}\right) .
\end{aligned}
$$

Since

$$
\begin{gathered}
\log H_{0, T}-\log \eta_{T}^{\lambda_{1}^{*}, \lambda_{2}} \\
=-\int_{0}^{T} r_{s} d s-\frac{1}{2} \int_{0}^{T}\left|\tilde{\lambda}_{s}-\theta_{s}\right|^{2} d s \\
-\int_{0}^{T}\left(\tilde{\lambda}_{s}-\theta_{s}\right) d B_{s}^{\lambda_{1}^{*}, \lambda_{2}},
\end{gathered}
$$

we have

$$
\begin{gathered}
Z_{t}^{\pi^{*}, \lambda_{1}^{*}, \lambda_{2}}=E^{P^{\lambda_{1}^{*}, \lambda_{2}}}\left[\left(\frac{y H_{0, T}}{\eta_{T}^{\lambda_{1}^{*}, \lambda_{2}}}\right)^{2} I^{\prime}\left(\frac{y H_{0, T}}{\eta_{T}^{\lambda_{1}^{*}, \lambda_{2}}}\right)\right. \\
\left(-\int_{t}^{T} D_{t}^{\lambda_{1}, \lambda_{2}} r_{s} d s-\left(\tilde{\lambda}_{t}-\theta_{t}\right)-\frac{1}{2} \int_{t}^{T} D_{t}^{\lambda_{1}, \lambda_{2}}\left|\tilde{\lambda}_{s}-\theta_{s}\right|^{2} d u\right. \\
\left.\left.-\int_{t}^{T} D_{t}^{\lambda_{1}, \lambda_{2}}\left(\tilde{\lambda}_{s}-\theta_{s}\right) d B_{s}^{\lambda_{1}^{*}, \lambda_{2}}\right) \mid \mathcal{F}_{t}^{\lambda_{1}^{*}, \lambda_{2}}\right] .
\end{gathered}
$$

Since $r \equiv 0$, the result follows.

For the exponential utility case with $r \equiv 0$, the result follows from $I^{\prime}(y)=-\frac{1}{p y}$ and $\frac{d Q}{d P^{\lambda_{1}^{*}, \lambda_{2}}}=\left(\frac{H_{0, T}}{\eta_{T}^{\lambda_{1}^{*}, \lambda_{2}}}\right)$. 


\section{B. Proposition 1 and Corollary 2}

In the following, we calculate $\psi$ for a general stochastic $r$ case. By ClarkOcone formula (e.g. Theorem 4.1 in Nunno et al. [6]),

$$
\psi_{t}=E^{Q}\left[D_{t}^{Q} \tilde{X}_{T}^{\pi^{*}} \mid \mathcal{F}_{t}^{Q}\right]
$$

We calculate $D_{t}^{Q} \tilde{X}_{T}^{\pi^{*}}$ as follows.

$$
\begin{gathered}
D_{t}^{Q} \tilde{X}_{T}^{*}=D_{t}^{Q}\left(I\left(\frac{y H_{0, T}}{\eta_{T}^{\lambda_{1}^{*}, \lambda_{2}^{*}}}\right) \exp \left(-\int_{0}^{T} r_{s} d s\right)\right) \\
=-I^{\prime}\left(\frac{y H_{0, T}}{\eta_{T}^{\lambda_{1}^{*}, \lambda_{2}^{*}}}\right) \frac{y H_{0, T}}{\eta_{T}} \exp \left(-\int_{0}^{T} r_{s} d s\right) \\
\quad \times D_{t}^{Q}\left(\log \eta_{T}^{\lambda_{1}^{*}, \lambda_{2}^{*}}-\log H_{0, T}\right) \\
+I\left(\frac{y H_{0, T}}{\eta_{T}^{\lambda_{1}^{*}, \lambda_{2}^{*}}}\right) \exp \left(-\int_{0}^{T} r_{s} d s\right)\left(-\int_{t}^{T} D_{t}^{Q} r_{s} d s\right) .
\end{gathered}
$$

Here, we used $\frac{y H_{0, T}}{\eta_{T}^{\lambda_{1}^{*}, \lambda_{2}^{*}}}=\exp \left(\log \left(\frac{y H_{0, T}}{\eta_{T}^{\lambda_{1}^{*}, \lambda_{2}^{*}}}\right)\right)$.

Since

$$
\begin{gathered}
\log \eta_{T}^{\lambda_{1}^{*}, \lambda_{2}^{*}} \\
=-\frac{1}{2} \int_{0}^{T}\left|\lambda_{s}^{*}\right|^{2} d s+\int_{0}^{T} \lambda_{s}^{* \top} \theta_{s} d s+\int_{0}^{T} \lambda_{s}^{* \top} d B_{s}^{Q},
\end{gathered}
$$

and

$$
\log H_{0, T}=-\int_{0}^{T} r_{s} d s+\frac{1}{2} \int_{0}^{T}\left|\theta_{s}\right|^{2} d s+\int_{0}^{T} \theta_{s}^{\top} d B_{s}^{Q},
$$

we have

$$
\begin{gathered}
\log \eta_{T}^{\lambda_{1}^{*}, \lambda_{2}^{*}}-\log H_{0, T} \\
=\int_{0}^{T} r_{s} d s-\frac{1}{2} \int_{0}^{T}\left|\lambda_{s}^{*}-\theta_{s}\right|^{2} d s+\int_{0}^{T}\left(\lambda_{s}^{*}-\theta_{s}\right)^{\top} d B_{s}^{Q} .
\end{gathered}
$$

Thus,

$$
\begin{gathered}
D_{t}^{Q}\left(\log \eta_{T}^{\lambda_{1}^{*}, \lambda_{2}^{*}}-\log H_{0, T}\right) \\
=\int_{t}^{T} D_{t}^{Q} r_{s} d s+\left(\lambda_{t}^{*}-\theta_{t}\right) \\
-\frac{1}{2} \int_{t}^{T} D_{t}^{Q}\left|\lambda_{s}^{*}-\theta_{s}\right|^{2} d s+\int_{t}^{T} D_{t}^{Q}\left(\lambda_{s}^{*}-\theta_{s}\right) d B_{s}^{Q},
\end{gathered}
$$

and

$$
\begin{gathered}
E^{Q}\left[D_{t}^{Q} \tilde{X}_{T}^{*} \mid \mathcal{F}_{t}^{Q}\right] \\
=E^{Q}\left[-I^{\prime}\left(\frac{y H_{0, T}}{\eta_{T}^{\lambda_{1}^{*}, \lambda_{2}^{*}}}\right) \frac{y H_{0, T}}{\eta_{T}} \exp \left(-\int_{0}^{T} r_{s} d s\right)\right. \\
\times\left(\int_{t}^{T} D_{t}^{Q} r_{s} d s+\left(\lambda_{t}^{*}-\theta_{t}\right)\right. \\
\left.-\frac{1}{2} \int_{t}^{T} D_{t}^{Q}\left|\lambda_{s}^{*}-\theta_{s}\right|^{2} d s+\int_{t}^{T} D_{t}^{Q}\left(\lambda_{s}^{*}-\theta_{s}\right) d B_{s}^{Q}\right) \\
\left.+I\left(\frac{y H_{0, T}}{\eta_{T}^{\lambda_{1}^{*}, \lambda_{2}^{*}}}\right) \exp \left(-\int_{0}^{T} r_{s} d s\right)\left(-\int_{t}^{T} D_{t}^{Q} r_{s} d s\right) \mid \mathcal{F}_{t}^{Q}\right] .
\end{gathered}
$$

Since $r \equiv 0$, the result follows.

In this appendix, we provide details of the sup-sup-inf problem in Examples 1 and 2 in Section VI, where it is solved concretely for the exponential utility. For the cases with a $\log$ utility, see Appendix D in the full version of this paper [22].

In the following, we consider an exponential utility case where

$$
u(x)=-\frac{1}{p} \exp (-p x), p>0
$$

and $I(y)=-\frac{\log y}{p}$.

Suppose that for given $\lambda_{2} \in \tilde{\Lambda}_{2}$, the saddle point $\left(\pi^{*}\left(\lambda_{2}\right), \lambda_{1}^{*}\left(\lambda_{2}\right)\right)$ that attains $\sup _{\pi \in \mathcal{A}} \inf _{\lambda_{1} \in \Lambda_{1}}$ is obtained.

Then, by (6), $X_{T}^{\pi^{*}}$ is expressed as

$$
X_{T}^{\pi^{*}}=-\frac{1}{p} \log \left(\frac{y H_{0, T}}{\eta_{T}^{\lambda_{1}^{*}, \lambda_{2}}}\right),
$$

where

$$
y=\exp \left(-p x-E^{Q}\left[\log H_{0, T}-\log \eta_{T}^{\lambda_{1}^{*}, \lambda_{2}}\right]\right) .
$$

Hence, we have

$$
\begin{gathered}
J\left(\pi^{*}, \lambda_{1}^{*}, \lambda_{2}\right)=E^{P^{\lambda_{1}^{*}, \lambda_{2}}}\left[u\left(X_{T}^{\pi^{*}}\right)\right] \\
=-\frac{y}{p} E^{P_{1}^{\lambda_{1}^{*}, \lambda_{2}}}\left[\frac{H_{0, T}}{\eta_{T}^{\lambda_{1}^{*}, \lambda_{2}}}\right]=-\frac{y}{p} \\
=-\frac{1}{p} \exp \left(-p x-\frac{1}{2} \int_{0}^{T} E^{Q}\left[\left(\lambda_{1, s}^{*}-\theta_{1, s}\right)^{2}\right.\right. \\
\left.\left.+\left(\lambda_{2, s}-\theta_{2, s}\right)^{2}\right] d s\right) .
\end{gathered}
$$

Particularly, if for any $\lambda_{2} \in \tilde{\Lambda}_{2}$, the saddle point $\left(\pi^{*}\left(\lambda_{2}\right), \lambda_{1}^{*}\left(\lambda_{2}\right)\right)$ is given by $\lambda_{1}^{*}=\bar{\lambda}_{1}, X_{T}^{\pi^{*}}=-\frac{1}{p} \log \left(\frac{y H_{0, T}}{\eta_{T}^{\lambda_{1}, \lambda_{2}}}\right)$ as we will observe in the following cases, the problem reduces to

$$
\begin{gathered}
\sup _{\lambda_{2} \in \tilde{\Lambda}_{2}} \frac{1}{2} \int_{0}^{T} E^{Q}\left[\left(\bar{\lambda}_{1, s}-\theta_{1, s}\right)^{2}+\left(\lambda_{2, s}-\theta_{2, s}\right)^{2}\right] d s \\
=\frac{1}{2} \int_{0}^{T} E^{Q}\left[\left(\bar{\lambda}_{1, s}-\theta_{1, s}\right)^{2}\right] d s \\
+\sup _{\lambda_{2} \in \tilde{\Lambda}_{2}} \frac{1}{2} \int_{0}^{T} E^{Q}\left[\left(\lambda_{2, s}-\theta_{2, s}\right)^{2}\right] d s .
\end{gathered}
$$

\section{Brownian case (exponential utility)}

Firstly, in the Brownian case where $\bar{\lambda}, \theta$ are proportional to a Brownian motion $B^{\bar{\lambda}_{1}, \bar{\lambda}_{2}}$ under $P^{\bar{\lambda}_{1}, \bar{\lambda}_{2}}$ as in Example 1 in Section VI-A, for any $\lambda_{2} \in \tilde{\Lambda}_{2},\left(\pi^{*}, \lambda_{1}^{*}\right)$ satisfying $\lambda_{1}^{*}=\bar{\lambda}_{1}$, and $X_{T}^{\pi^{*}}=$ $-\frac{1}{p} \log \left(\frac{y H_{0, T}}{\eta_{T}^{\lambda_{1}, \lambda_{2}}}\right)$ is a saddle point.

Since $\bar{\lambda}_{1}=\bar{\sigma}_{\lambda, 1} B_{1}^{\bar{\lambda}_{1}, \bar{\lambda}_{2}}, \lambda_{2}=\sigma_{\lambda, 2} B_{2}^{\bar{\lambda}_{1}, \bar{\lambda}_{2}}$ and $\theta_{j}=\sigma_{\theta, j} B_{j}^{\bar{\lambda}_{1}}, \bar{\lambda}_{2}, j=1,2$, and $B_{j}^{\bar{\lambda}_{1}, \bar{\lambda}_{2}}$ is an OrnsteinUhlenbeck process of $B_{j}^{\bar{\lambda}_{1}, \lambda_{2}}, B_{1, t}^{\bar{\lambda}_{1}, \bar{\lambda}_{2}}=B_{1, t}^{\bar{\lambda}_{1}, \lambda_{2}}, B_{2, t}^{\bar{\lambda}_{1}, \bar{\lambda}_{2}}=$ $\int_{0}^{t} e^{-\left(\bar{\sigma}_{\lambda, 2}-\sigma_{\lambda, 2}\right)(t-s)} d B_{2, s}^{\bar{\lambda}_{1}, \lambda_{2}}$, thus $X_{T}^{\pi^{*}}=-\frac{1}{p} \log \left(\frac{y H_{0, T}}{\eta_{T}^{\lambda_{1}, \lambda_{2}}}\right)$ is $\mathcal{F}_{T}^{\bar{\lambda}_{1}, \lambda_{2}}$-measurable and in particular in $\mathbf{D}_{1,1}^{\bar{\lambda}_{1}, \lambda_{2}}$. Thus, we can apply Corollary 1.

By Corollary 1, noting that $r=0$,

$$
\begin{gathered}
D_{1, t}^{\bar{\lambda}_{1}, \lambda_{2}}\left(\bar{\lambda}_{1, s}-\theta_{1, s}\right)=\left(\bar{\sigma}_{\lambda, 1}-\sigma_{\theta, 1}\right) 1_{\{s \geq t\}} \\
D_{1, t}^{\bar{\lambda}_{1}, \lambda_{2}}\left(\bar{\lambda}_{1, s}-\theta_{1, s}\right)^{2}=2\left(\bar{\sigma}_{\lambda, 1}-\sigma_{\theta, 1}\right)^{2} B_{1, s}^{\bar{\lambda}_{1}, \bar{\lambda}_{2}} 1_{\{s \geq t\}}, \\
\frac{d Q}{d P^{\bar{\lambda}_{1}, \lambda_{2}}}=\frac{H_{0, T}}{\eta_{T}^{\bar{\lambda}_{1}, \lambda_{2}}},
\end{gathered}
$$

and $B_{t}^{Q}=B_{t}^{\bar{\lambda}_{1}, \lambda_{2}}-\int_{0}^{t}\left(\theta_{s}-\tilde{\lambda}_{s}\right) d s$ is a $\left\{\mathcal{F}_{t}^{\bar{\lambda}_{1}, \lambda_{2}}\right\}$-Brownian motion under $Q$, we calculate

$$
Z_{1, t}^{\bar{\lambda}_{1}, \lambda_{2}}=\frac{1}{p}\left(\frac{y H_{0, t}}{\eta_{t}^{\bar{\lambda}_{1}, \lambda_{2}}}\right)\left(\bar{\sigma}_{\lambda, 1}-\sigma_{\theta, 1}\right) B_{1, t}^{\bar{\lambda}_{1}, \bar{\lambda}_{2}} .
$$

Since

$$
\bar{\sigma}_{\lambda, 1}-\sigma_{\theta, 1}>0, \bar{\sigma}_{\lambda, 1}<0
$$


we have

$$
\operatorname{sgn}\left(Z_{1, t}^{\pi^{*}, \lambda_{1}^{*}, \lambda_{2}}\right)=\operatorname{sgn}\left(B_{1, t}^{\bar{\lambda}_{1}, \bar{\lambda}_{2}}\right)=-\operatorname{sgn}\left(\bar{\lambda}_{1, t}\right),
$$

and

$$
-\left|\bar{\lambda}_{1, t}\right| \operatorname{sgn}\left(Z_{1, t}^{\pi^{*}, \lambda_{1}^{*}, \lambda_{2}}\right)=\bar{\lambda}_{1, t}=\lambda_{1, t}^{*} .
$$

Thus, (6) and (8) in (i),(ii) in Section IV are satisfied and $\lambda_{1}^{*}=\bar{\lambda}_{1}$ and $\pi^{*}$ generating $X_{T}^{\pi^{*}}=-\frac{1}{p} \log \left(\frac{y H_{0, T}}{\eta_{T}^{\lambda_{1}, \lambda_{2}}}\right)$ provide a saddle point.

Then, the problem reduces to

$$
\begin{gathered}
\quad \frac{1}{2} \int_{0}^{T} E^{Q}\left[\left(\bar{\lambda}_{1, s}-\theta_{1, s}\right)^{2}\right] d s \\
+\sup _{\lambda_{2} \in \tilde{\Lambda}_{2}} \frac{1}{2} \int_{0}^{T} E^{Q}\left[\left(\lambda_{2, s}-\theta_{2, s}\right)^{2}\right] d s \\
=\frac{1}{2} \int_{0}^{T} E^{Q}\left[\left(\bar{\lambda}_{1, s}-\theta_{1, s}\right)^{2}\right] d s \\
+\sup _{-\bar{\sigma}_{\lambda, 2} \leq \sigma_{\lambda, 2} \leq \bar{\sigma}_{\lambda, 2}} \frac{1}{2}\left(\sigma_{\lambda, 2}-\sigma_{\theta, 2}\right)^{2} \int_{0}^{T} E^{Q}\left[\left(B_{2, s}^{\bar{\lambda}_{1}, \bar{\lambda}_{2}}\right)^{2}\right] d s .
\end{gathered}
$$

Here,

$$
B_{2, s}^{\bar{\lambda}_{1}, \bar{\lambda}_{2}}=\int_{0}^{s} e^{-\left(\bar{\sigma}_{\lambda, 2}-\sigma_{\theta, 2}\right)(s-u)} d B_{2, u}^{Q}
$$

and

$$
\begin{gathered}
\int_{0}^{T} E^{Q}\left[\left(B_{2, s}^{\bar{\lambda}_{1}, \bar{\lambda}_{2}}\right)^{2}\right] d s \\
=\int_{0}^{T} \int_{0}^{s} e^{-2\left(\bar{\sigma}_{\lambda, 2}-\sigma_{\theta, 2}\right)(s-u)} d u d s<\infty .
\end{gathered}
$$

Since $\sigma_{\theta, 2} \leq 0$, the supremum is attained at

$$
\sigma_{\lambda, 2}^{*}=\bar{\sigma}_{\lambda, 2} \text {. }
$$

\section{Square-root case (exponential utility)}

Next, in the case where $\bar{\lambda}$ and $\theta$ are proportional to a square-root of a square-root process as in Example 2 in Section VI-C, for any $\lambda_{2} \in \tilde{\Lambda}_{2}$, $\left(\pi^{*}, \lambda_{1}^{*}\right)$ satisfying $\lambda_{1}^{*}=\bar{\lambda}_{1}$ and $X_{T}^{\pi^{*}}=-\frac{1}{p}\left(\frac{y H_{0, T}}{\eta_{T}^{\lambda_{1}, \lambda_{2}}}\right)$ is a saddle point.

Firstly,

$$
\begin{gathered}
D_{j, t}^{\bar{\lambda}_{1}, \lambda_{2}} V_{j, s}=D_{j, t}^{\bar{\lambda}_{1}, \lambda_{2}}\left(\sqrt{V_{j, s}}\right)^{2} \\
=2 \sqrt{V_{j, s}} D_{j, t}^{\bar{\lambda}_{1}, \lambda_{2}} \sqrt{V_{j, s}}
\end{gathered}
$$

where

$$
\begin{gathered}
D_{j, t}^{\bar{\lambda}_{1}, \lambda_{2}} \sqrt{V_{j, s}}= \\
\frac{\sigma_{v, j}}{2} \exp \left(\int_{t}^{s}\left[-\frac{\kappa_{j}}{2}-\frac{\left(\frac{\kappa_{j} \bar{\theta}_{j}}{2}-\frac{\sigma_{v, j}^{2}}{8}\right)}{V_{j, u}}\right] d u\right)>0
\end{gathered}
$$

by Proposition 4.1 in Alos and Ewald [1] and $\sigma_{v, j}>0$.

Since $\bar{\lambda}_{1}=\bar{\sigma}_{\lambda, 1} \sqrt{V_{1}}, \lambda_{2}=\sigma_{\lambda, 2} \sqrt{V_{2}}$ and $\theta_{j}=\sigma_{\theta, j} \sqrt{V_{j}}, j=1,2$, and $V_{j}$ is a square-root process, which is expressed as a unique strong solution of SDE

$$
\begin{gathered}
d V_{1, t}=\kappa_{1}\left(\bar{\theta}_{1}-V_{1, t}\right) d t+\sigma_{v, 1} \sqrt{V_{1, t}} d B_{1, t}^{\bar{\lambda}_{1}, \lambda_{2}} \\
d V_{2, t} \\
=\left(\kappa_{2}\left(\bar{\theta}_{2}-V_{2, t}\right)-\sigma_{v, 2}\left(\bar{\sigma}_{\lambda, 2}-\sigma_{\lambda, 2}\right) V_{2, t}\right) d t+\sigma_{v, 2} \sqrt{V_{2, t}} d B_{2, t}^{\bar{\lambda}_{1}, \lambda_{2}} \\
V_{j, 0}>0, j=1,2,
\end{gathered}
$$

thus $X_{T}^{\pi^{*}}=-\frac{1}{p} \log \left(\frac{y H_{0, T}}{\eta_{T}^{\lambda_{1}, \lambda_{2}}}\right)$ is $\mathcal{F}_{T}^{\bar{\lambda}_{1}, \lambda_{2}}$-measurable and in particular in $\mathbf{D}_{1,1}^{\bar{\lambda}_{1}, \lambda_{2}}$. Thus, we can apply Corollary 1 .
By Corollary 1, noting that $r=0$,

$$
\begin{gathered}
D_{1, t}^{\bar{\lambda}_{1}, \lambda_{2}}\left(\bar{\lambda}_{1, s}-\theta_{1, s}\right)=\left(\bar{\sigma}_{\lambda, 1}-\sigma_{\theta, 1}\right) D_{1, t}^{\bar{\lambda}_{1}, \lambda_{2}}\left(\sqrt{V_{1, s}}\right), \\
D_{1, t}^{\bar{\lambda}_{1}, \lambda_{2}}\left(\bar{\lambda}_{1, s}-\theta_{1, s}\right)^{2}=2\left(\bar{\sigma}_{\lambda, 1}-\sigma_{\theta, 1}\right)^{2} \sqrt{V_{1, s}} D_{1, t}^{\bar{\lambda}_{1}, \lambda_{2}}\left(\sqrt{V_{1, s}}\right), \\
\frac{d Q}{d P^{\bar{\lambda}_{1}, \lambda_{2}}}=\frac{H_{0, T}}{\eta_{T}^{\bar{\lambda}_{1}, \lambda_{2}}},
\end{gathered}
$$

and $\int_{t}^{T} D_{1, t}^{\bar{\lambda}_{1}, \lambda_{2}}\left(\sqrt{V_{1, s}}\right) d B_{s}^{\bar{\lambda}_{1}, \lambda_{2}}$ is a $\left\{\mathcal{F}_{t}^{\bar{\lambda}_{1}, \lambda_{2}}\right\}$-martingale under $P^{\bar{\lambda}_{1}, \lambda_{2}}$, we calculate

$$
Z_{1, t}^{\bar{\lambda}_{1}, \lambda_{2}}=\frac{1}{p}\left(\frac{y H_{0, t}}{\eta_{t}^{\bar{\lambda}_{1}, \lambda_{2}}}\right)\left(\bar{\sigma}_{\lambda, 1}-\sigma_{\theta, 1}\right) \sqrt{V_{1, t}} .
$$

Since $\bar{\sigma}_{\lambda, 1}-\sigma_{\theta, 1}>0$, we have $\operatorname{sgn}\left(Z_{1, t}^{\pi^{*}, \lambda_{1}^{*}, \lambda_{2}}\right)=+1=\operatorname{sgn}\left(\bar{\lambda}_{1, t}\right)$ and

$$
\begin{gathered}
-\left|\bar{\lambda}_{1, t}\right| \operatorname{sgn}\left(Z_{1, t}^{\pi^{*}, \lambda_{1}^{*}, \lambda_{2}}\right)=-\left|\bar{\lambda}_{1, t}\right| \operatorname{sgn}\left(\bar{\lambda}_{1, t}\right) \\
=\bar{\lambda}_{1, t}=\lambda_{1, t}^{*} .
\end{gathered}
$$

Thus, (6) and (8) in (i),(ii) in Section IV are satisfied, and $\lambda_{1}^{*}=\bar{\lambda}_{1}$ and $\pi^{*}$ generating $X_{T}^{\pi^{*}}=-\frac{1}{p} \log \left(\frac{y H_{0, T}}{\eta_{T}^{\lambda_{1}, \lambda_{2}}}\right)$ provide a saddle point.

Then, the problem reduces to

$$
\begin{gathered}
\quad \frac{1}{2} \int_{0}^{T} E^{Q}\left[\left(\bar{\lambda}_{1, s}-\theta_{1, s}\right)^{2}\right] d s \\
+\sup _{\lambda_{2} \in \tilde{\Lambda}_{2}} \frac{1}{2} \int_{0}^{T} E^{Q}\left[\left(\lambda_{2, s}-\theta_{2, s}\right)^{2}\right] d s \\
=\frac{1}{2} \int_{0}^{T} E^{Q}\left[\left(\bar{\lambda}_{1, s}-\theta_{1, s}\right)^{2}\right] d s \\
+\sup _{-\bar{\sigma}_{\lambda, 2} \leq \sigma_{\lambda, 2} \leq \bar{\sigma}_{\lambda, 2}} \frac{1}{2}\left(\sigma_{\lambda, 2}-\sigma_{\theta, 2}\right)^{2} \int_{0}^{T} E^{Q}\left[V_{2, s}^{2}\right] d s .
\end{gathered}
$$

Here, $V_{2}$ satisfies the SDE

$$
\begin{gathered}
d V_{2, t} \\
=\left(\kappa_{2}\left(\bar{\theta}_{2}-V_{2, t}\right)-\sigma_{v, 2}\left(\bar{\sigma}_{\lambda, 2}-\sigma_{\theta, 2}\right) V_{2, t}\right) d t \\
+\sigma_{v, 2} \sqrt{V_{2, t}} d B_{2, t}^{Q} \\
V_{2,0}>0,
\end{gathered}
$$

and

$$
\int_{0}^{T} E^{Q}\left[V_{2, s}^{2}\right] d s \leq T E^{Q}\left[\sup _{0 \leq s \leq T} V_{2, s}^{2}\right]<\infty .
$$

Since $\sigma_{\theta, 2} \leq 0$, the supremum is attained at

$$
\sigma_{\lambda, 2}^{*}=\bar{\sigma}_{\lambda, 2}
$$

